\title{
ASURAT: functional annotation-driven unsupervised clustering of single-cell transcriptomes
}

Keita Iida ${ }^{1, *}$, Jumpei Kondo ${ }^{2,3}$, Johannes Nicolaus Wibisana ${ }^{1}$, Masahiro Inoue ${ }^{3}$, Mariko Okada $^{1,4}$

\footnotetext{
${ }^{1}$ Institute for Protein Research, Osaka University, Suita, Osaka 565-0871, Japan

${ }^{2}$ Division of Health Sciences, Osaka University Graduate School of Medicine, Suita, Osaka 565-0871, Japan

${ }^{3}$ Department of Clinical Bio-resource Research and Development, Graduate School of Medicine Kyoto University, Kyoto 606-8501, Japan

${ }^{4}$ Center for Drug Design and Research, National Institutes of Biomedical Innovation, Health and Nutrition, Ibaraki, Osaka, 567-0085, Japan

*Correspondence: kiida@protein.osaka-u.ac.jp
} 


\begin{abstract}
Motivation: Single-cell RNA sequencing (scRNA-seq) analysis reveals heterogeneity and dynamic cell transitions. However, conventional gene-based analyses require intensive manual curation to interpret the biological implications of computational results. Hence, a theory for efficiently annotating individual cells is necessary.

Results: We present ASURAT, a computational pipeline for simultaneously performing unsupervised clustering and functional annotation of disease, cell type, biological process, and signaling pathway activity for single-cell transcriptomic data, using correlation graph-based decomposition of genes based on database-derived functional terms. We validated the usability and clustering performance of ASURAT using scRNAseq datasets for human peripheral blood mononuclear cells, which required fewer manual curations than existing methods. Moreover, we applied ASURAT to scRNA-seq and spatial transcriptome datasets for small cell lung cancer and pancreatic ductal adenocarcinoma, identifying previously overlooked subpopulations and differentially expressed genes. ASURAT is a powerful tool for dissecting cell subpopulations and improving biological interpretability of complex and noisy transcriptomic data.
\end{abstract}

Availability: A GPLv3-licensed implementation of ASURAT is on GitHub (https://github.com/keita-iida/ASURAT). 


\section{Introduction}

Single-cell RNA sequencing (scRNA-seq) has deepened our knowledge of biological complexity in terms of heterogeneity and dynamic transition of cell populations in a variety of phenomena, and this knowledge has immense potential for elucidating the regulatory principles underlying our body plans (La Manno, et al., 2018). scRNA-seq has been widely used to improve the molecular understanding of malignant cells in lymphoma (Zhang, et al., 2019), intra- and intertumoral heterogeneity in drug-treated cancer populations (Stewart, et al., 2020), ligand-receptor interaction in tumor immune microenvironments (Chen, et al., 2020), and the effects of viral infection on immune cell populations (Devitt, et al., 2019). Various clustering methods based on gene expression similarity have been proposed (Pasquini, et al., 2021) and applied to annotate cell types (Kim, et al., 2020). However, conventional gene-based analyses require intensive manual curation to annotate clustering results; hence, efficient and unbiased interpretation of single-cell data remains challenging (Andrews, et al., 2021; Aran, et al., 2019; Gao, et al., 2019; Kiselev, et al., 2019; Lahnemann, et al., 2020).

Conventionally, single-cell transcriptomes are analyzed and interpreted by means of unsupervised clustering followed by manual curation of marker genes chosen from a large number of differentially expressed genes (DEGs) (Andrews, et al., 2021; Lahnemann, et al., 2020). Here, manual curations are based on literature searches of biological functions of DEGs. Today, several computational tools for semi-automated cell type and marker gene inference based on clustering results are available to assist manual annotation, as detailed in the review by Pasquini et al. (2021). However, this is often difficult because a single gene is generally multifunctional and therefore associated with multiple biological function terms (Cancer Genome Atlas Research, et al., 2017). In cancer transcriptomics, this difficulty is exacerbated by the complex interdependence between disease-related biomarker genes and their heterogeneous expressions, which are associated with numerous biological function terms.

A possible solution is to realize cell clustering and biological interpretation at the same time. Recently, reference component analysis (RCA), which is used for accurate clustering of single-cell transcriptomes along with unbiased cell-type annotation based on 
similarity to reference transcriptome panels ( $\mathrm{Li}$, et al., 2017). Yet, these methods require the transcriptomic data of well-characterized reference cells as learning datasets, which might not always be available. Another approach is using supervised classification (Gao, et al., 2019) combined with gene set enrichment analysis, incorporating biological knowledge and functions; hence, it may improve the interpretability over signature genebased approaches, which place sole emphasis on individual roles of genes (Fan, et al., 2016). Despite these advances, we still lack a prevailing theory leveraging this information at the single-cell level.

To overcome the aforementioned limitations, a method providing simultaneous interpretation of biological function and classification of the cells is needed for singlecell analysis. Thus, we propose an original computational pipeline named ASURAT (functional annotation-driven unsupervised clustering of single-cell transcriptomes), which simultaneously performs unsupervised cell clustering and biological interpretation in terms of cell type, disease, biological process, and signaling pathway activity. In this study, we demonstrate the clustering performance of ASURAT using standard scRNAseq and spatial transcriptome (ST) datasets for human peripheral blood mononuclear cells (PBMCs), small cell lung cancer (SCLC), and pancreatic ductal adenocarcinoma (PDAC), respectively. We show that ASURAT can greatly improve functional understanding of single-cell transcriptomes, adding a new layer of biological interpretability to conventional gene-based analyses.

\section{Methods}

\section{Overview of ASURAT workflow}

ASURAT was developed to simultaneously cluster and interpret single-cell transcriptomes using functional gene sets (FGSs) (Figure 1), and it was implemented in the R programming language. FGSs are collected from knowledge-based databases (DBs) for disease, cell type, biological process, and signaling pathway activity, such as Disease Ontology (DO) (Yu, et al., 2015), Cell Ontology (CO) (Diehl, et al., 2016), Gene Ontology (GO) (Yu, et al., 2012), and Kyoto Encyclopedia of Genes and Genomes (KEGG) (Kanehisa and Goto, 2000) by implementing R packages such as DOSE (version 3.16.0), ontoProc (version 1.12.0), clusterProfiler (version 3.18.0), and 
KEGGREST (version 1.30.0), respectively (Figure 1b). Then, ASURAT created multiple biological terms using single-cell transcriptome data and the FGSs (Figure 1c, Supplementary Note 1). We called such new biological terms signs. Finally, ASURAT created a sign-by-sample matrix (SSM), in which rows and columns stand for signs and samples (cells), respectively (Figure 1c). SSM is analogous to a read count table, where the rows represent signs with biological meaning instead of individual genes and that the values contained are sign scores instead of read counts. By analyzing SSMs, individual cells can be characterized by various biological terms (Figure 1d).

\section{Sign}

Let $A$ be a read count table of size $p \times n$ from single-cell transcriptomic data, whose rows and columns mean $p$ genes, represented by $\Omega=\{1,2, \cdots, p\}$, and $n$ cells, respectively, and $R$ a "relation" (e.g., correlation matrix) among $\Omega$. Let $\mathcal{F}=$ $\left\{\left(T_{k}, \Omega_{k}\right) \mid k=1,2, \cdots, q\right\}$ be a set of ordered pairs, where $T_{k}$ and $\Omega_{k} \in 2^{\Omega}$ ( $2^{\Omega}$ is a power set of $\Omega$ ) are biological description and the FGS, respectively. Consider an $R$ dependent representation $\Omega_{k}=\bigcup_{j=1}^{m_{k}} \Omega_{k}^{(j)}$, where $m_{k}$ is an integer, for $k=1,2, \cdots, q$. Then, the triplet $\left(T_{k}, \Omega_{k}^{(j)}, R\right)$ is termed a sign, in particular $\left(T_{k}, \Omega_{k}, R\right)$ a parent sign. Our definition is inspired by Saussure's semiology as described in the early 20th century. According to Maruyama (2008), the original notion of a signe is a segment of a thing of interest, which is created by an arbitrary decomposition based on its relationships. For example, a rainbow is a continuum of varying light input, from which we can see distinct colors of red, yellow, green, and blue by our subjective decomposition based on their spectral relationships (Couper, 2015).

\section{Correlated gene set}

Let $R=\left(r_{i, j}\right)$ be a correlation matrix of size $p \times p$ defined by $A$ and a certain measure (e.g., Pearson's measure), whose diagonal elements are 1. Let $\alpha$ and $\beta$ be positive and negative constants satisfying $0<\alpha \leq 1$ and $-1 \leq \beta<0$, respectively. Let us arbitrarily fix $\left(T_{k}, \Omega_{k}\right) \in \mathcal{F}$ and consider the following subsets of $\Omega_{k}$ :

$$
\begin{aligned}
& U_{k}(\alpha)=\left\{i \in \Omega_{k} \mid \exists j \in \Omega_{k} \text { such that } r_{i, j} \geq \alpha, i \neq j\right\}, \\
& V_{k}(\beta)=\left\{i \in \Omega_{k} \mid \exists j \in \Omega_{k} \text { such that } r_{i, j} \leq \beta, i \neq j\right\},
\end{aligned}
$$




$$
W_{k}(\alpha, \beta)=U_{k}(\alpha) \cup V_{k}(\beta)
$$

Hereinafter we omit the arguments $\alpha$ and $\beta$ for simplicity. Let us denote $\Omega_{k}^{(\mathrm{w})}=\Omega_{k}-$ $W_{k}$. If $V_{k}$ is not empty, represent each element of $W_{k}$ as a point in the Euclidean space spanned by the row vectors of $R$ and decompose $W_{k}$ into two disjoint subsets by Partitioning Around Medoids (PAM) clustering (Schubert and Rousseeuw, 2019), that is $W_{k}=\Omega_{k}^{(\mathrm{s})} \cup \Omega_{k}^{(\mathrm{v})}$. Otherwise, if $V_{k}$ is empty, let $\Omega_{k}^{(\mathrm{s})}=U_{k}$ and $\Omega_{k}^{(\mathrm{v})}=\phi$ (empty). Thus $\Omega_{k}$ is decomposed into three parts as follows:

$$
\Omega_{k}=\Omega_{k}^{(\mathrm{s})} \cup \Omega_{k}^{(\mathrm{v})} \cup \Omega_{k}^{(\mathrm{w})}
$$

Let $\mu_{k}^{(\mathrm{s})}$ (resp. $\mu_{k}^{(\mathrm{v})}$ ) be the mean of off-diagonal elements of $R$ for $\Omega_{k}^{(\mathrm{s})}\left(\Omega_{k}^{(\mathrm{v})}\right)$, and assume $\mu_{k}^{(\mathrm{s})} \geq \mu_{k}^{(\mathrm{v})}$ without loss of generality. If $\mu_{k}^{(\mathrm{s})} \geq \alpha$, then $\Omega_{k}^{(\mathrm{s})}, \Omega_{k}^{(\mathrm{v})}$, and $\Omega_{k}^{(\mathrm{w})}$ are termed strongly, variably, and weakly correlated gene sets, respectively, which are hereafter abbreviated as SCG, VCG, and WCG. Otherwise, correlated gene sets cannot be defined for $T_{k}$.

For any given $\left(T_{k}, \Omega_{k}, R\right)$ the genes should strongly and positively correlate within each of the $\Omega_{k}^{(\mathrm{s})}$ and $\Omega_{k}^{(\mathrm{v})}$, while they should negatively correlate between $\Omega_{k}^{(\mathrm{s})}$ and $\Omega_{k}^{(\mathrm{v})}$. Thus, one can hypothesize that SCG and VCG are predominantly associated with $T_{k}$, which may aid interpretation of biological meanings of corresponding signs. Figure 2 shows that the SCG and VCG include KRT18 and ASCL1, which respectively have negative and positive contributions for lung small cell carcinoma. Thus, we interpret that $\left(T_{k}, \Omega_{k}^{(\mathrm{s})}, R\right)$ and $\left(T_{k}, \Omega_{k}^{(\mathrm{v})}, R\right)$ for DOID 5409 relate positively and negatively with this cell type, respectively.

Though simpler methods decomposing correlation graphs exist, such as one-shot PAM clustering (Schubert and Rousseeuw, 2019), hierarchical clustering and tree cutting (Murtagh and Legendre, 2014), principal component analysis (PCA)-based methods (Hyvarinen, 1999), and several graph statistical approaches (Blondel, et al., 2008; Bodenhofer, et al., 2011), we found that our VCG definition is critical for clustering cells. In fact, we tried replacing our decomposition method (1) with one-shot PAM 
clustering, but the results frequently exhibited deteriorated performance because both VCG and WCG (obtained from the one-shot clustering) included many weakly correlated genes.

\section{Sign-by-sample matrix}

Let $A=\left(a_{i, j}\right)$ be a gene-by-cell matrix of size $p \times n$ from a single-cell transcriptomic data, whose entries stand for normalized-and-centered gene expression levels. For simplicity, let us assume that functional gene sets $\Omega_{k}$ can be decomposed into non-empty $\Omega_{k}^{(\mathrm{s})}, \Omega_{k}^{(\mathrm{v})}$, and $\Omega_{k}^{(\mathrm{w})}$, for $k=1,2, \cdots, q$. Let $B^{(x)}, x \in\{\mathrm{s}, \mathrm{v}, \mathrm{w}\}$, be matrices of size $q \times n$, whose entries $b_{k, j}^{(x)}$ are defined as follows:

$$
b_{k, j}^{(x)}=\frac{1}{\left|\Omega_{k}^{(x)}\right|} \sum_{i \in \Omega_{k}^{(x)}} a_{i, j}
$$

where $\left|\Omega_{k}^{(x)}\right|$ stands for the number of elements in $\Omega_{k}^{(x)}$. Additionally, let $C^{(x)}, x \in\{\mathrm{s}, \mathrm{v}\}$, be $q \times n$ matrices as follows:

$$
C^{(x)}=\omega^{(x)} B^{(x)}+\left(1-\omega^{(x)}\right) B^{(\mathrm{w})},
$$

where $\omega^{(x)}, 0 \leq \omega^{(x)} \leq 1$, are weight constants. Here $C^{(\mathrm{s})}$ and $C^{(\mathrm{v})}$ are termed sign-bysample matrices (SSMs) for SCG and VCG, respectively, and the entry $c_{k, j}^{(x)}$ a sign score of the $k$ th sign and $j$ th sample (cell). By vertically concatenating SSMs for SCGs and VCGs, we created a single SSM. Note that ensemble means of sign scores across cells are zeros because SSMs are derived from the centered gene expression matrix $A$.

\section{Unsupervised clustering of sign-by-sample matrices}

One focus of analyzing SSMs is to cluster cells and find significant signs (Figure 1d), where "significant" means that the sign scores, i.e., the entries of (2), are specifically upregulated or downregulated at the cluster level. It should be noted that significant signs are analogous to DEGs but bear biological meanings. Here, naïve usages of statistical tests and fold change analyses should be avoided because the row vectors of 
SSMs are centered. Hence, we propose a nonparametric separation index, which quantifies the extent of separation between two sets of random variables (Supplementary Note 2). To cluster cells, we used two strategies. The first is unsupervised clustering, such as PAM, hierarchical, and graph-based clustering. The second is a method of extracting a continuous tree-like topology using diffusion map (Coifman and Lafon, 2006), followed by allocating cells to different branches of the data manifolds (Parra, et al., 2019). Choosing an appropriate strategy depends on the biological context, but the latter is usually applied to developmental processes or timecourse experimental data, which are often followed by pseudotime analyses.

\section{Results}

\section{Clustering single-cell transcriptomes of peripheral blood mononuclear cells}

To validate the usability and clustering performance of ASURAT in comparison with the existing methods, we analyzed two public scRNA-seq datasets, namely the PBMC $4 \mathrm{k}$ and $6 \mathrm{k}$ datasets (Supplementary Note 3 ), in which the cell types were inferred using computational tools based on prior assumptions (Cao, et al., 2020). We first excluded low-quality genes and cells and attenuated technical biases with respect to zero inflation and variation of capture efficiencies between cells using bayNorm (Tang, et al., 2020) (Supplementary Note 4). The resulting read count tables were supplied to ASURAT and four other methods: scran (version 1.18.7) (Lun, et al., 2016), Seurat (version 4.0.2) (Hao, et al., 2021), Monocle 3 (version 1.0.0) (Trapnell, et al., 2014), and SC3 (version 1.18.0) (Kiselev, et al., 2017). To infer existing cell types and the population ratios in the PBMC 4k and 6k datasets, we implemented the existing methods using settings close to the default ones, performed cell clustering, and annotated each cluster by manually investigating DEGs based on the false discovery rates $($ FDRs $)<10^{-99}$ (Figure 3a, Supplementary Note 5). When using ASURAT, we performed unsupervised cell clustering and semi-automatic annotation based on SSMs for CO, GO, and KEGG.

Among all the existing methods, Seurat and Monocle 3 could robustly reproduce most blood cell type labels, as inferred by Cao et al. (2020), while scran and SC3 output many unspecified cells (Figure 3c). We found that the Seurat pipeline, followed by manual annotations based only on a couple of DEGs, provided comparable population 
ratios with previous results (Cao, et al., 2020). However, it was quite laborious to manually select marker genes from numerous DEGs (Figure 3a), which tend to increase in terms of the number of cells as well as significance levels. Based on the clustering results of Seurat, we assigned the labels (i) T cell, (ii) monocyte, (iii) B cell, and (iv) NK or NKT cell to the cells in PBMC 4k (resp. PBMC 6k) by finding marker genes from (i) 57 (114), (ii) 102 (148), (iii) 49 (33), and (iv) 32 (35) DEGs, respectively. To avoid such a laborious process, it is possible to implement automatic annotation tools based on the calculated DEGs, such as by using scCATCH (version 2.1) (Shao, et al., 2020). Nevertheless, population ratios inferred by Seurat with scCATCH were less consistent than those by Seurat with manual annotations (Figure 3c).

ASURAT simultaneously performed unsupervised cell clustering and biological interpretation leveraging all defined FGSs, without relying on DEGs (Figure 3a). We identified five cell type labels, with none remaining unspecified (Figure 3b, c, Figure $\mathrm{S} 2$ ). The population ratios were approximately consistent with the reported values (Cao, et al., 2020), except for the small dendritic cell population possibly included in PBMCs (Villani, et al., 2017; Wagner, 2018). Such a small discrepancy was unavoidable, because Cao et al. (2020) used author-defined DEGs and preselected cell types to identify the most preferable ones. Unexpectedly, the clustering results using SSMs for GO and KEGG also showed well-separated clusters in two-dimensional Uniform Manifold Approximation and Projection (UMAP) (McInnes and Healy, 2018) spaces (Figure S3), indicating that the functional states of cells are also heterogeneous with respect to biological process and signaling pathway activity. These results demonstrate that ASURAT can perform robust clustering for single-cell transcriptomes.

\section{Clustering a small cell lung cancer single-cell transcriptome}

SCLC tumors undergo a transition from chemosensitivity to chemoresistance states against platinum-based therapy (Stewart, et al., 2020). Stewart et al. (2020) analyzed scRNA-seq data obtained from circulating tumor cell-derived xenografts generated from treatment-naïve lung cancer patients, cultured them with vehicle or cisplatin treatments, and reported that the gene expression profiles of the platinum-resistant tumors were more heterogeneous than those of platinum-sensitive tumors. However, the mechanism 
behind chemoresistance remains unclear, partly because transcriptional heterogeneity is affected by physiological states of cells such as pathological states (Stewart, et al., 2020), cell cycle (Dominguez, et al., 2016), and metabolic processes (Jalili, et al., 2021), which cannot be readily identified by conventional marker gene-based analyses alone. To better understand SCLC subtypes in chemoresistant tumors, we applied Seurat and ASURAT to the published SCLC scRNA-seq data (Supplementary Note 3) (Stewart, et al., 2020).

First, we investigated the expression levels of known SCLC marker genes (Ireland, et al., 2020), namely ASCL1, NEUROD1, YAP1, and POU2F3 and confirmed that almost all of the cells are of the ASCL1 single-positive subtype (Figure S4), which is consistent with the previous report (Stewart, et al., 2020). After quality controls, the data were normalized by bayNorm (Tang, et al., 2020) and the resulting read count table was supplied to the workflows of Seurat and ASURAT (Supplementary Note 4, 6). To investigate molecular subtypes and potential resistance pathways, we clustered the single-cell transcriptome and inferred a cell cycle phase for each cell using Seurat (Hao, et al., 2021), as shown in the UMAP spaces (Figure 4e). We found that the cell populations assigned to $\mathrm{G} 1, \mathrm{~S}$, and G2M phases are sequentially distributed in the UMAP space, indicating that the clustering results are considerably affected by the cell cycle. Then, we identified DEGs for each cluster (Group 1, 2, and 3) and performed KEGG enrichment analysis using clusterProfiler (Yu, et al., 2012), but the chemoresistance terms were not primarily enriched (Figure $\mathbf{4 f}$ ).

Subsequently, to investigate functional heterogeneities in SCLCs, we used ASURAT to create SSMs using DO, GO, and KEGG. Based on the SSM for DO, we performed a dimensionality reduction using diffusion map (Coifman and Lafon, 2006), which showed a tree-like topology. Then, we defined a pseudotime along the branches and clustered the single-cell transcriptome using MERLoT (Parra, et al., 2019) (Figure 4c). Based on pseudotime analysis, we revealed that sign scores for platinum drug resistance (path:hsa01524_S) and PD-L1 expression-mediated immunosuppression (path:hsa05235_S) were upregulated in clusters 2 and 3, respectively. In addition, sign scores for intracellular protein transport (GO:0006886_S), with an FGS including the 
SCLC malignancy marker CD24 (Kristiansen, et al., 2003), was upregulated in cluster 1 (Figure 4d). We noticed that sign scores for hematopoietic system disease (DOID:74_S) were moderately increased in cluster 1 (separation index 0.38), which was supported by a previous work reporting that hematopoietic cancers are similar to SCLCs in terms of gene expression profiles and drug sensitivities (Balanis, et al., 2019). Although the SCLC molecular subtypes have been extensively studied (Chen, et al., 2019; Ireland, et al., 2020; Schwendenwein, et al., 2021; Wooten, et al., 2019; Yatabe, 2020), data regarding the functional subtypes of ASCL1-positive SCLC remain limited. To identify de novo SCLC subtypes, future work will validate our clustering results.

Finally, we vertically concatenated all the SSMs, cell cycle phases, and expression matrices to characterize individual cells from multiple biological aspects, as shown by the heatmaps along with the clustering result of ASURAT (Figure 4a, b). As shown, we were able to simultaneously perform unsupervised clustering and biological interpretation of single-cell transcriptomes. Moreover, we added a layer of DEGs using multiple Mann-Whitney $U$ tests (Figure 4a), showing that most DEGs had been previously overlooked (Chen, et al., 2019; Ireland, et al., 2020; Schwendenwein, et al., 2021; Wooten, et al., 2019; Yatabe, 2020). Taken together, we provide a novel clue for the clinical improvements for relapsed SCLC tumors.

\section{Clustering a pancreatic ductal adenocarcinoma spatial transcriptome}

Moncada et al. (2020) analyzed scRNA-seq and ST data obtained from PDAC patients (Moncada, et al., 2020) and reported that cancer and non-cancer cells are spatially distributed in the distinct tissue regions of the primary PDAC tumors, and that PDAC cells are accompanied by inflammatory fibroblasts. Since the cellular resolutions of the STs were estimated to be 20-70 cells per ST spot, which is far lower than that of scRNA-seq, computational methods have been proposed to predict existing cell types by integrating ST and scRNA-seq datasets (Elosua-Bayes, et al., 2021; Moncada, et al., 2020). Here, we aimed to dissect ST data and compare the annotation results of ASURAT with those of Seurat by using ST (PDAC-A ST1) and scRNA-seq (PDAC-A inDrop from 1 to 6) datasets (Moncada, et al., 2020) (Supplementary Note 3). 
First, we combined all the scRNA-seq datasets after confirming that there were minimal batch effects (Figure S5). Then, the ST and scRNA-seq data were normalized by bayNorm (Tang, et al., 2020) (Supplementary Note 4) and the resulting read count tables were supplied to Seurat. To cluster the ST with reference to the scRNA-seq data, we performed canonical correlation analysis (CCA)-based data integration of Seurat (Figure 5a), followed by an unsupervised clustering of the integrated transcriptome using Seurat functions, which is shown in UMAP spaces (Figure 5b) and the tissue image (Figure 5c). Unexpectedly, batch effects were not corrected between ST and scRNA-seq datasets after data integration; nevertheless, the inferred cancer and noncancer regions were approximately consistent with previously annotated histological regions (Elosua-Bayes, et al., 2021; Moncada, et al., 2020), wherein several marker genes such as REG1A, S100A4 and TM4SF1, and CELA2A were identified as DEGs for clusters 2, 3, and 5, respectively (FDRs $<10^{-80}$, Mann-Whitney $U$ tests).

Next, we input the ST and scRNA-seq integrated transcriptome into ASURAT workflow. To investigate complex PDAC tissues, we created SSMs using DO, CO, GO, and KEGG, as well as CellMarker (Zhang, et al., 2019) and MSigDB (Subramanian, et al., 2005). Based on the SSM for GO, which was computed from the integrated transcriptome, we performed a dimensionality reduction using PCA and clustered the SSM by $k$-nearest neighbor (KNN) graph generation and the Louvain algorithm, which is shown in UMAP spaces (Figure 5d) and the tissue image (Figure 5e). Remarkably, ASURAT was able to remove the aforementioned batch effects and infer the spots we suspect as atypical region which might be a normal pancreas involved in cancer (Figure 5e left bottom).

To further investigate cell states in these spots, we computed all the sign scores across the tissue (Figure S6). We found that the sign scores for PDAC (DOID:3498_S), which has an FGS including PDAC markers such as $S 100 P$ and $M M P 1$, were increased in the ST spots approximately matching the reported PDAC region (Moncada, et al., 2020), while those for transcriptional misregulation in cancer (path:hsa05202_S) and microRNAs in cancer (path:hsa05206_S) were increased both in the previously annotated PDAC spots and the newly predicted atypical spots (Figure 5f). These newly 
predicted spots were also annotated by a sign for Th17 cell differentiation (path:hsa04659_S), suggesting tumor-associated inflammation or antitumor immunity through intercellular communications between Th17 and cancer cells (MullerHubenthal, et al., 2009), which remains to be elucidated in PDAC (Liu, et al., 2019).

It is reported that in more than $90 \%$ of PDAC cases, $K R A S$ is mutated at the $\mathrm{G}$ domain of the 12th residue (Ischenko, et al., 2021; Luchini, et al., 2020). Hence, we speculated that it might be possible to validate our clustering results of cancer and non-cancer spots by comparing the frequencies of KRAS mutations using ST data. Unfortunately, we were unable to detect any read mapped to the specific reported region, possibly owing to the shallow read depth and inherent $3^{\prime}$ bias present in the data. We hope that simultaneous genetic and transcriptional profiling can address this problem in the future (Lee, et al., 2020).

\section{Discussion}

We have developed ASURAT, a novel computational pipeline for simultaneous cell clustering and biological interpretation using FGSs. ASURAT begins by performing a correlation graph-based decomposition of FGS to define multiple biological terms, termed signs. ASURAT then transforms scRNA-seq data into an SSM, whose rows and columns stand for signs and samples, respectively. This SSM plays a key role in characterizing individual cells by various biological terms. Applying ASURAT to several scRNA-seq and spatial transcriptome datasets for PBMCs, SCLC, and PDAC, we robustly reproduced the previously reported blood cell types, identified putative subtypes of chemoresistant SCLC, and identified distinct regions within the PDAC tissue.

Conventionally, single-cell transcriptomes are analyzed and interpreted by means of unsupervised clustering followed by manual curation of marker genes chosen from a large number of DEGs, which has been a common bottleneck of gene-based analyses (Andrews, et al., 2021; Aran, et al., 2019; Gao, et al., 2019). The statistical significance of individual genes, typically defined by $p$-value or fold change, is dependent on clustering results, which are also affected by various physiological states of cells 
(Dominguez, et al., 2016; Jalili, et al., 2021). Here, we expect that ASURAT provides an alternative approach using FGSs and demonstrates superior performance for identifying functional subtypes even within a fairly homogeneous population such as isolated cancer cells. In practice, complemental usages of ASURAT and existing methods (Butler, et al., 2018; La Manno, et al., 2018) will provide more comprehensive understanding of single-cell and spatial transcriptomes, helping us shed light on putative transdifferentiation of neuroendocrine cancers (Balanis, et al., 2019; Kubota, et al., 2020), intercellular communication in tumor immune microenvironments (Maynard, et al., 2020), and virus infection on immune cell populations (Devitt, et al., 2019).

In omics data analyses, knowledge-based DBs are used to interpret computational results: GO, KEGG pathway, and motif enrichment analyses are often used for transcriptomic and epigenomic analyses (McLeay and Bailey, 2010; Mootha, et al., 2003; Reimand, et al., 2019). In contrast, we propose a unique analytical workflow, in which such DBs are used for simultaneous clustering and biological interpretation by defining signs from single-cell transcriptome data and FGSs. This framework is potentially applicable to any multivariate data with variables linked with annotation information. We can also find such datasets in studies of $\mathrm{T}$ cell receptor sequencing (De Simone, et al., 2018; Rempala, et al., 2011) along with a pan-immune repertoire (Zhang, et al., 2020). We anticipate that ASURAT will make it possible to identify various intersample differences among $\mathrm{T}$ cell receptor repertoires in terms of cellular subtype, antigen-antibody interaction, genetic and pathological backgrounds.

Finally, future challenges in data-driven mathematical analysis are worth noting. Since ASURAT can create multivariate data (i.e., SSMs) from multiple signs, ranging from cell types to biological functions, it will be valuable to consider graphical models of signs, from which we may infer conditional independence structures. A non-Gaussian Markov random field theory (Morrison, et al., 2017) is one of the most promising approaches to this problem, but it requires quite a large number of samples for achieving true graph edges (Morrison, et al., 2017). As available data expand in size and diversity, biological interpretation will become increasingly important. Hence, future work should improve methods for prioritizing biological terms more efficiently than 
manual screening. We hope ASURAT will greatly facilitate our intuitive understanding of various biological data and open new means of general functional annotation-driven data analysis.

\section{Acknowledgements}

We thank Dr. Takeya Kasukawa for comments that improved the analysis pipeline.

\section{Funding}

K.I. was supported by JSPS KAKENHI Grant No. 20K14361. K.I., J.K., and M.I. were supported by Shin Bunya Kaitaku Shien Program of Institute for Protein Research, Osaka University. M.O. was supported by JSPS KAKENHI Grant No. 17H06299, 17H06302, and 18H04031, JST-Mirai program No. JPMJMI19G7, and JST CREST Program No. JPMJCR21N3. M.O. and M.I. were supported by P-CREATE, Japan Agency for Medical Research and Development. M.O. and K.I. were supported by JST Moonshot R\&D Grant Number JPMJMS2021.

\section{Data Availability statement}

The PBMCs datasets are available in the 10x Genomics repository at https://support.10xgenomics.com/single-cell-gene-expression/datasets. The SCLC and PDAC datasets are available in Gene Expression Omnibus with accession codes GSE138474 (GSM4104164) and GSE111672 (GSM3036909, GSM3036910, GSM3036911, GSM3405527, GSM3405528, GSM3405529, and GSM3405530), which are referenced in (Stewart, et al., 2020) and (Moncada, et al., 2020), respectively.

Conflicts of Interest: none declared. 
a Flowchart for ASURAT

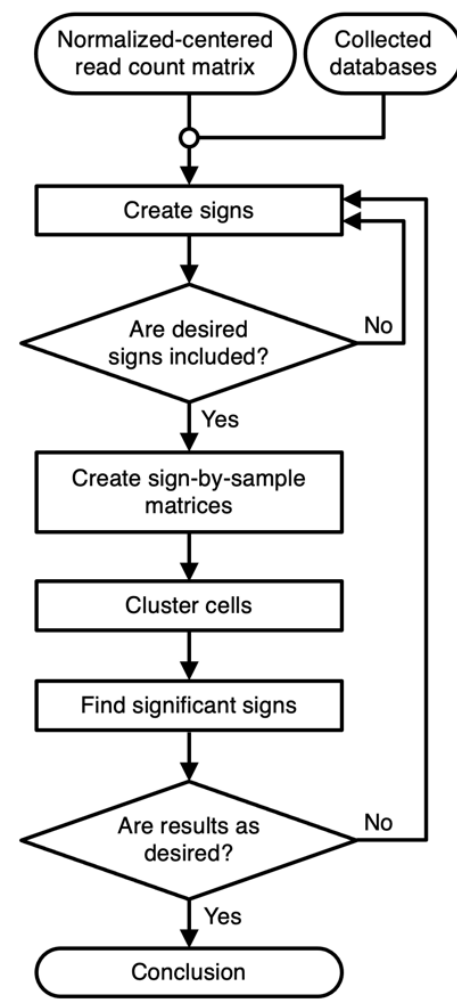

b Collect databases

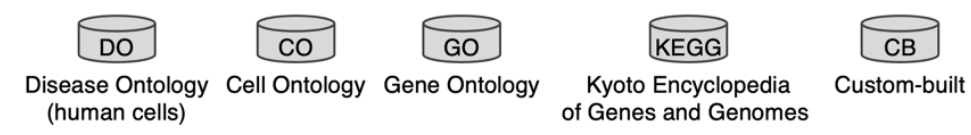

c

Classification of genes into signs using established databases
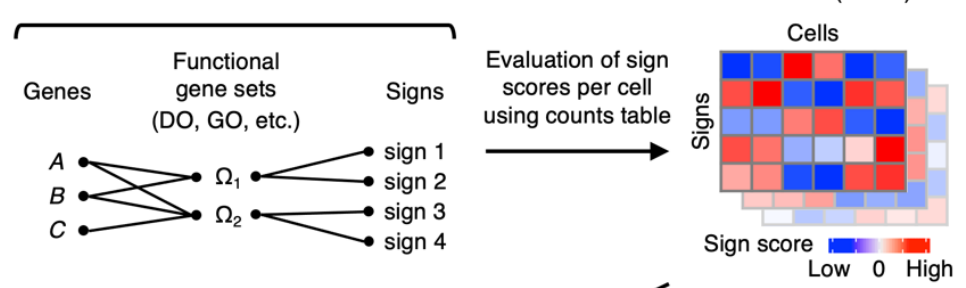

d Analyze SSMs
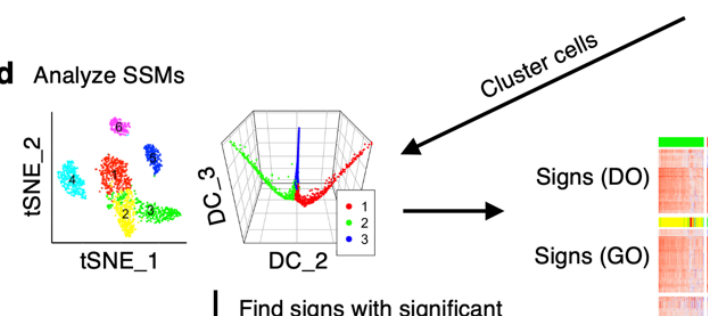
Low 0 High

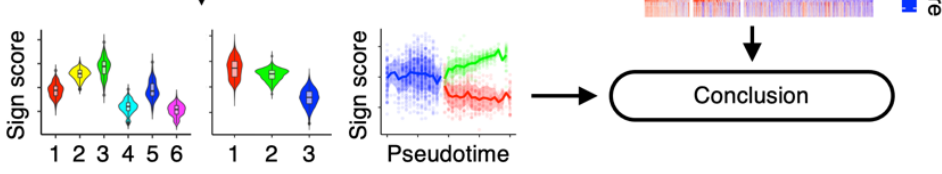

Figure 1. Workflow of ASURAT. (a) Flowchart of the procedures. (b) Collection of knowledge-based data-bases (DBs). (c) Creation of sign-by-sample matrices (SSMs) from normalized-and-centered read count table and the collected DBs. (d) Analysis of SSMs to infer diseases, cell types, biological processes, and signaling pathway activities. 


\begin{tabular}{|c|c|c|c|c|}
\hline Description & \multicolumn{4}{|c|}{$T_{k}=$ "Lung small cell carcinoma" } \\
\hline Gene set & $\begin{array}{c}\Omega_{k}=\{K R T 18, A S C L 1, \\
T P 53, \ldots\}\end{array}$ & $\begin{array}{l}\Omega_{k}^{(\mathrm{s})}=\{K R T 18, \\
C D 9, L G A L S 3\}\end{array}$ & $\begin{array}{l}\Omega_{k}^{(v)}=\{I G F B P 2, \\
A S C L 1, C A L C A\}\end{array}$ & $\begin{array}{c}\Omega_{k}^{(w)}=\{T P 53, \\
R B 1, V E G F A, \ldots\}\end{array}$ \\
\hline $\begin{array}{l}\text { Correlation } \\
\text { graph }\end{array}$ & (1) & & & (176) \\
\hline
\end{tabular}

Figure 2. Representation of correlation graph-based decomposition. From single-cell RNA sequencing data and a Disease Ontology (DO) term with DOID 5409, which concerns small cell lung cancer, three signs $\left(T_{k}, \Omega_{k}^{(j)}, R\right), j \in\{\mathrm{s}, \mathrm{v}, \mathrm{w}\}$, were produced from their parent sign $\left(T_{k}, \Omega_{k}, R\right)$ by decomposing the correlation graph $\left(\Omega_{k}, R\right)$ into strongly, variably, and weakly correlated gene sets: $\Omega_{k}^{(\mathrm{s})}, \Omega_{k}^{(\mathrm{v})}$, and $\Omega_{k}^{(\mathrm{w})}$, respectively. Red and blue edges in correlation graphs indicate positive and negative correlations, respectively; color density indicates the strength of the correlation. 
Conventional scRNA-seq workflow (scran, Seurat, Monocle 3, SC3, etc.)

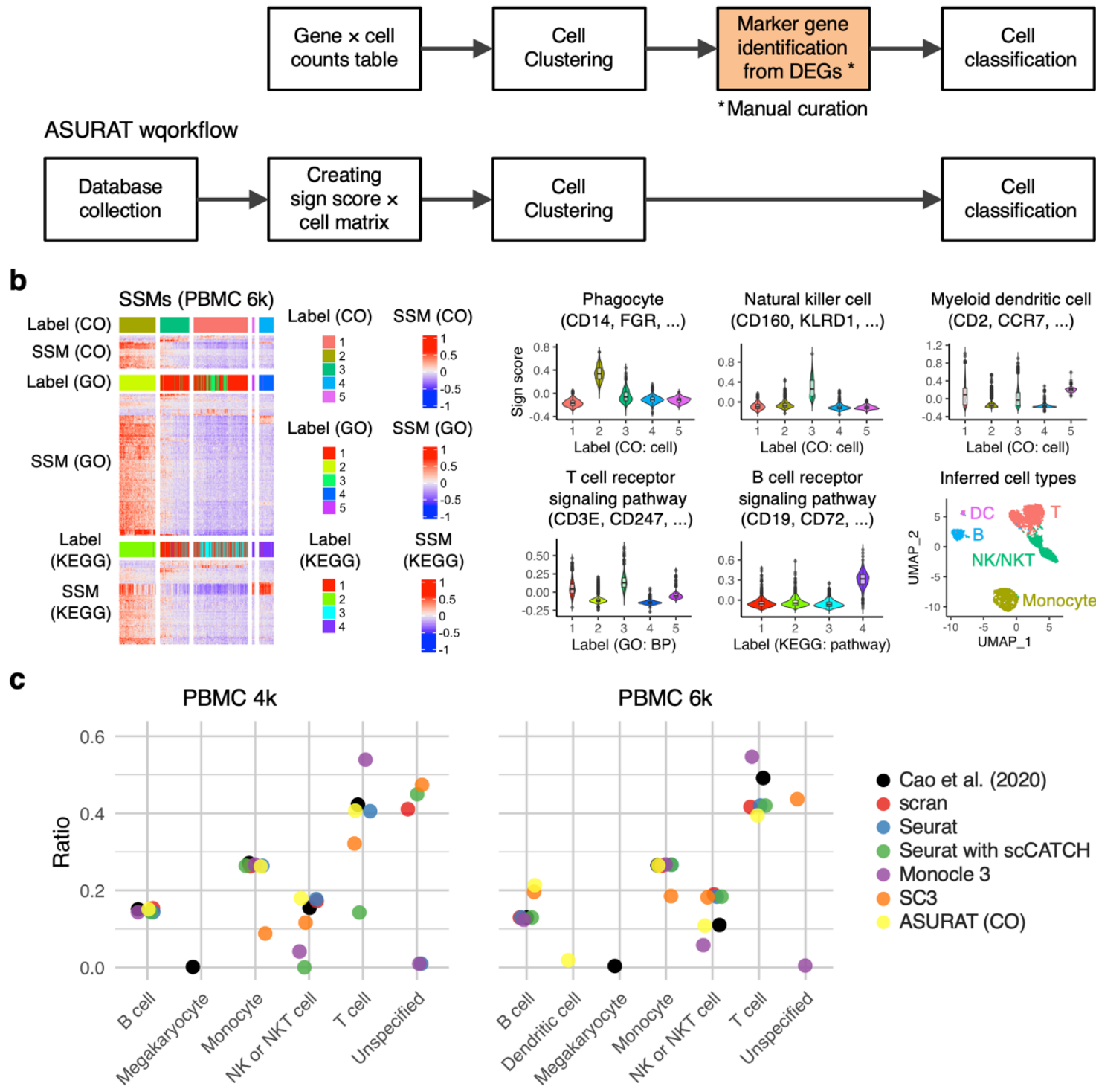

Figure 3. Clustering peripheral blood mononuclear cell (PBMC) single-cell transcriptomes. (a) Schematic illustration of conventional single-cell RNA sequencing and ASURAT workflows. (b) Identification of cell types in the PBMC 6k dataset from analyses of sign-by-sample matrices (SSMs) for Cell Ontology (CO), Gene Ontology (GO), and Kyoto Encyclopedia of Genes and Genomes (KEGG). According to heatmaps and violin plots of representative signs and functional gene sets, T cell (“T”), B cell ("B"), NK or NKT cell ("NK/NKT"), monocyte, and dendritic cell ("DC") were identified as shown in Uniform Manifold Approximation and Projection (UMAP) plots. 
(c) Population ratios in the PBMC 4k and 6k datasets predicted by seven different methods. DEG, differentially expressed gene.

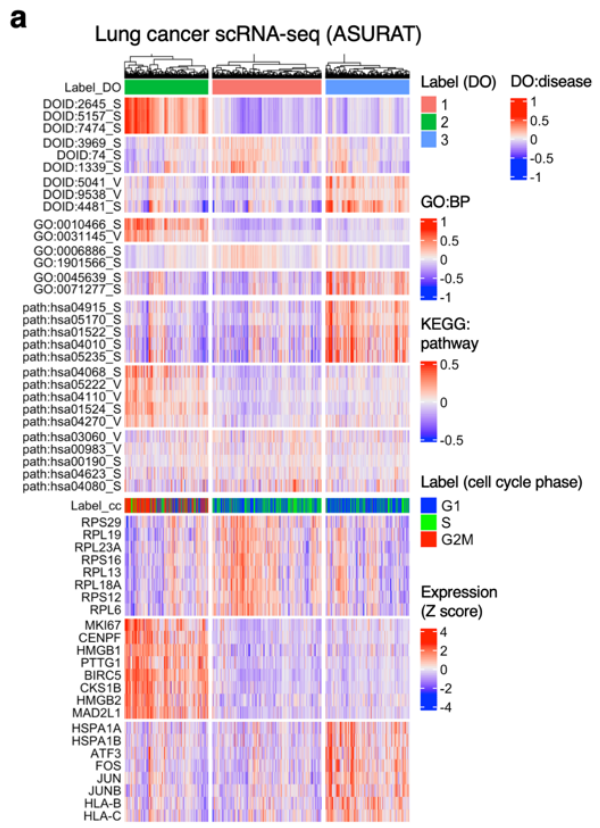

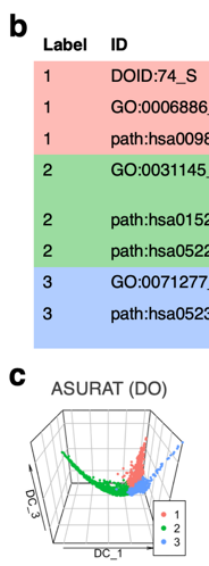

e

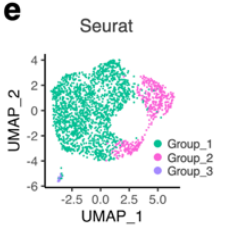

Description

Hescriptopoietic system disease
intracellular protein transport
Drug metabolism
Anaphase-promoting complex-dependent
catabolic process
Platinum drug resistance
Small cell lung cancer
Cellular response to calcium ion
PD-L1 expression and PD-1 checkpoint
pathway in cancer

d
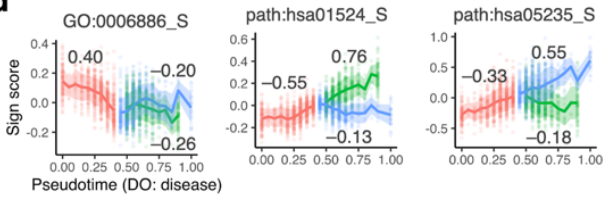

'

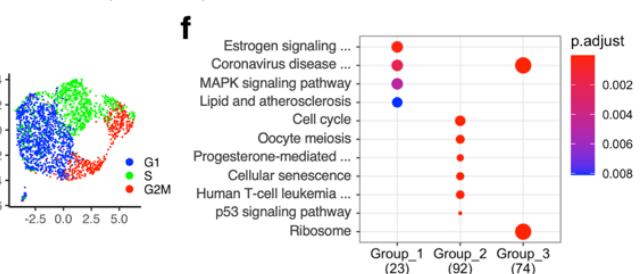

Functional gene set Category

CD24, MIF, ... DO CD24, SEC61G, ... GO MGST3, GSTO1, ... KEGG CDC20, CDK1,... GO

TOP2A, BIRC5, ... KEGG TP53, PTEN, ... KEGG JUN, FOS, NFKBIA, HIF1A, ... KEGG

235 _S

Figure 4. Clustering a single-cell transcriptome of small cell lung cancers. (a) Heatmaps showing (i) clustering results of ASURAT, (ii) sign scores of sign-by-sample matrices (SSMs) for Disease Ontology (DO), Gene Ontology (GO), and Kyoto Encyclopedia of Genes and Genomes (KEGG), and (iii) scaled gene expression levels, which are concatenated vertically. Here, only the most significant signs and differentially expressed genes (DEGs) for ASURAT clusters are shown. (b) Representative signs from (a). (c) Diffusion map of the SSM for DO, projected onto the first three coordinates. (d) Sign scores for the indicated IDs along the pseudotime, in which the standard deviations are shown by the shaded area. The value on each plot stands for the separation index for a given group versus all the others. The clustering labels are consistent with those in (a) and (b). (e) Clustering results and cell cycle phases computed by Seurat. (f) KEGG pathway enrichment analysis based on DEGs for Seurat clusters in (e). 
a
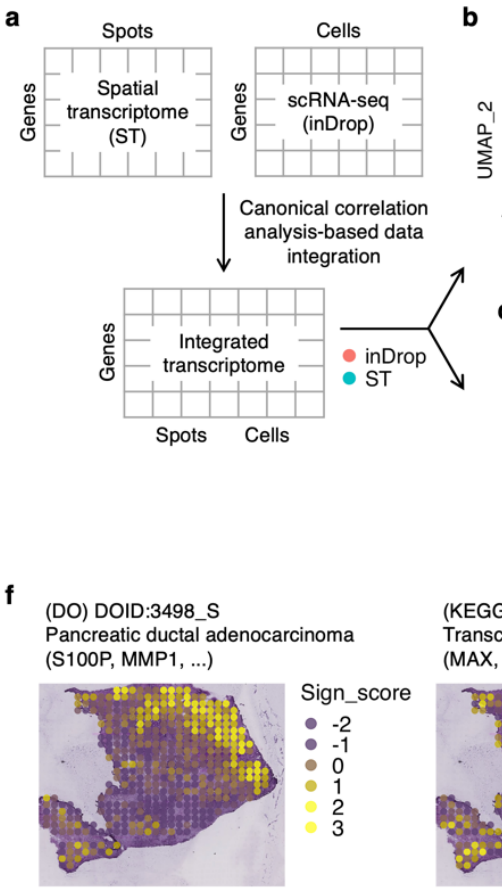

b

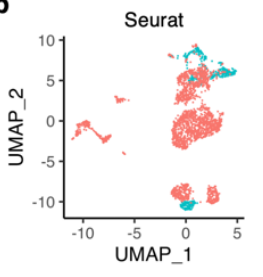

d asurat (GO)

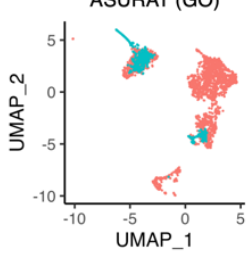

(KEGG) path:hsa05202_S Transcriptional misregulation in cancer (MAX, FOXO1, BIRC2, RXRA, ...)

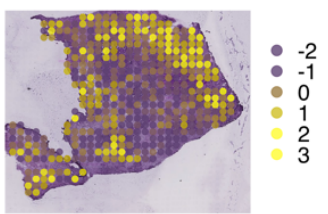

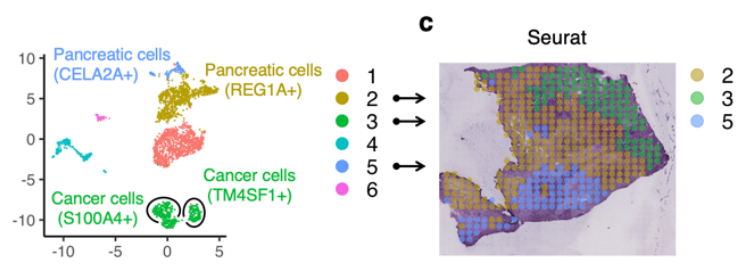

e asurat (GO)

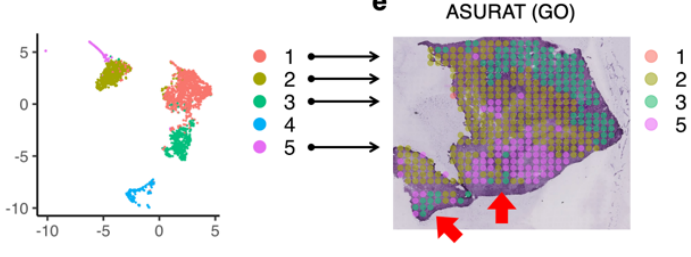

(KEGG) path:hsa05206_S MicroRNAs in cancer (VIM, PDCD4, FSCN1, ...)

(KEGG) path:hsa04659_S Th17 cell differentiation (CD4, HLA-DRA, RELA, RXRA, ...)

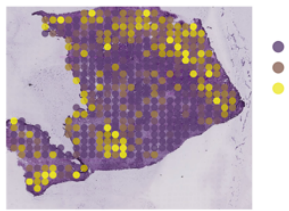

Figure 5. Clustering of spatial transcriptome (ST) data of pancreatic ductal adenocarcinoma (PDAC). (a) Canonical correlation analysis-based data integration of single-cell RNA sequencing (scRNA-seq) and ST datasets using Seurat. (b) Seurat unsupervised clustering based on the integrated data. Cells were manually labeled according to the indicated differentially expressed genes (DEGs) in Uniform Manifold Approximation and Projection (UMAP) plots. (e) ASURAT clustering result shown in the PDAC tissue, in which red arrows indicate the spots newly predicted as atypical region which might be a normal pancreas involved in cancer. (f) Profiles of sign scores in the PDAC tissue, predicting cancer and inflammation spots. DO, Disease Ontology. GO, Gene Ontology. KEGG, Kyoto Encyclopedia of Genes and Genomes. 


\section{Supplementary Materials}

Figure S1. Detailed workflow of Figure 1c focusing on the parameter settings

Figure S2. Clustering peripheral blood mononuclear cell (PBMC) 4k single-cell transcriptomes using ASURAT

Figure S3. Clustering peripheral blood mononuclear cell (PBMC) 4k and 6k single-cell transcriptomes using ASURAT

Figure S4. Heatmaps of expression levels of known small cell lung cancer marker genes

Figure S5. Data qualities across all the cells in single-cell RNA-seq datasets PDAC-A inDrop from 1 to 6

Figure S6. Sign scores for functions and signaling pathway activities using Gene Ontology (GO) and Kyoto Encyclopedia of Genes and Genomes (KEGG) across the PDAC tissue

Supplementary Note 1. Parameter settings of ASURAT

Supplementary Note 2. Separation index

Supplementary Note 3. Datasets

Supplementary Note 4. Data preprocessing: quality control, normalization, and centering

Supplementary Note 5. Analysis of scRNA-seq datasets of PBMC 4k and 6k

Supplementary Note 6. Analysis of an SCLC scRNA-seq dataset

Supplementary Note 7. Limitations of the study 


\section{Detailed workflow of Figure 1C}

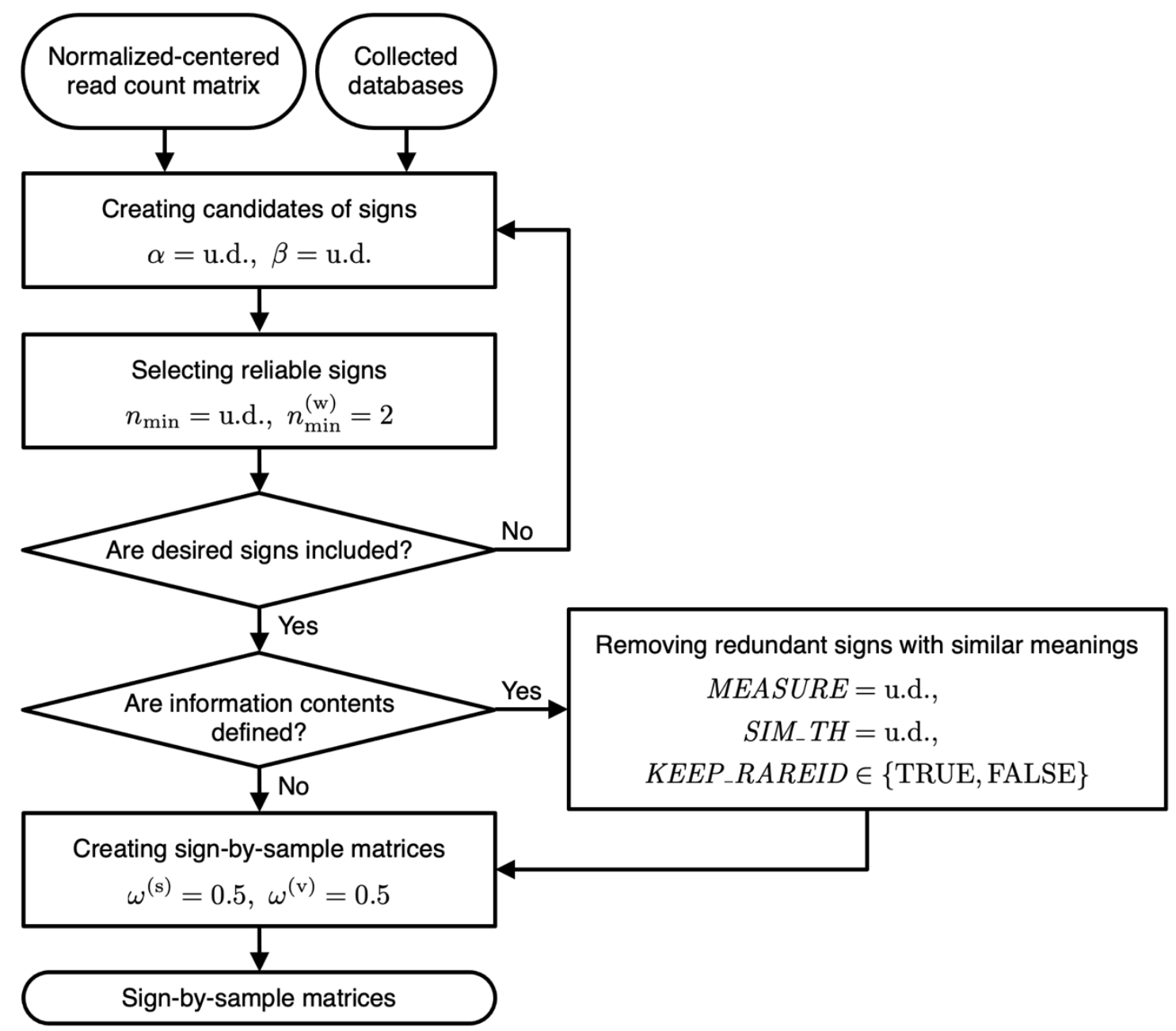

Figure S1. Detailed workflow of Figure 1c focusing on the parameter settings. The indicated values are preset as default in ASURAT, while "u.d." stands for the value or argument that users must define. Here, $\alpha$ and $\beta$ are positive and negative threshold values of correlation coefficients; $n_{\min }$ and $n_{\min }^{(\mathrm{w})}$, positive integers for selecting reliable signs; MEASURE, the name of information content (IC)-based method defining semantic similarities; SIM_TH, a threshold value used to regard two biological terms as similar; KEEP_RAREID determines whether the signs with larger ICs are kept or not (if TRUE, the signs with larger ICs are kept), and $\omega^{(\mathrm{s})}$ and $\omega^{(\mathrm{v})}$ weight constants are used to define sign-by-sample matrices. 


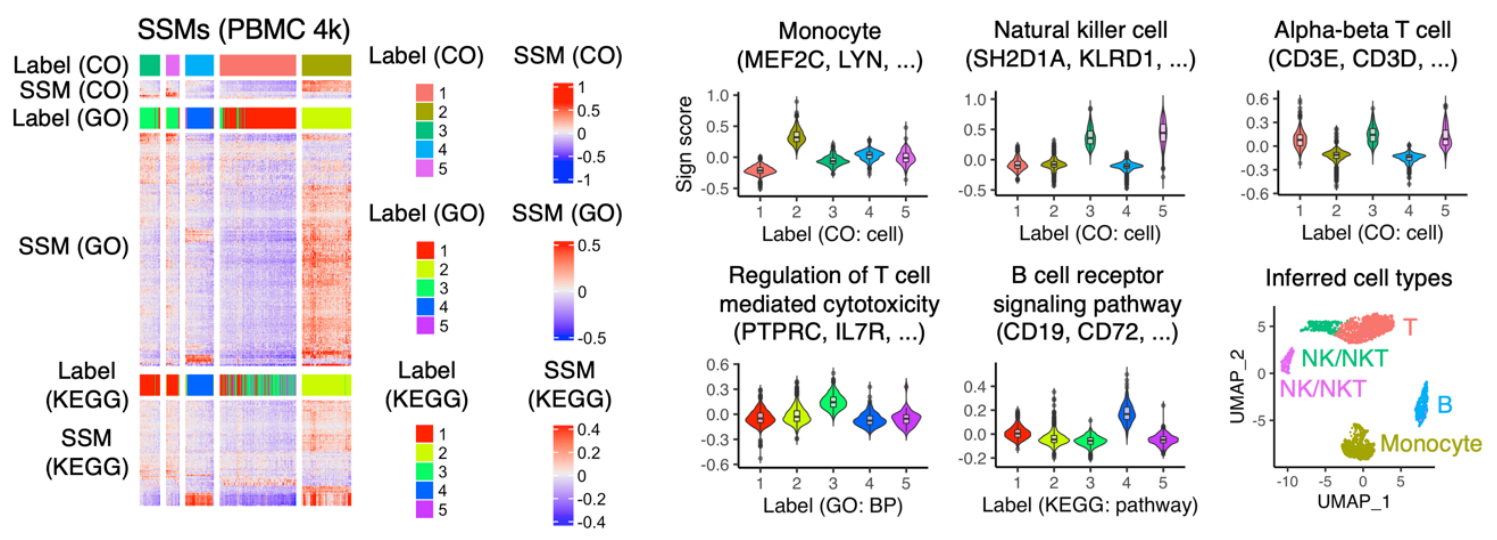

Figure S2. Clustering peripheral blood mononuclear cell (PBMC) 4k single-cell transcriptomes using ASURAT. Identification of cell types in the PBMC 4k dataset from analyses of sign-by-sample matrices (SSMs) for Cell Ontology (CO), Gene Ontology (GO), and Kyoto Encyclopedia of Genes and Genomes (KEGG). According to heatmaps and violin plots, showing representative signs and the functional gene sets, T cell (“T”), B cell (“B”), NK or NKT cell (“NK/NKT"), and monocyte were identified as shown in Uniform Manifold Approximation and Projection (UMAP) plots. 

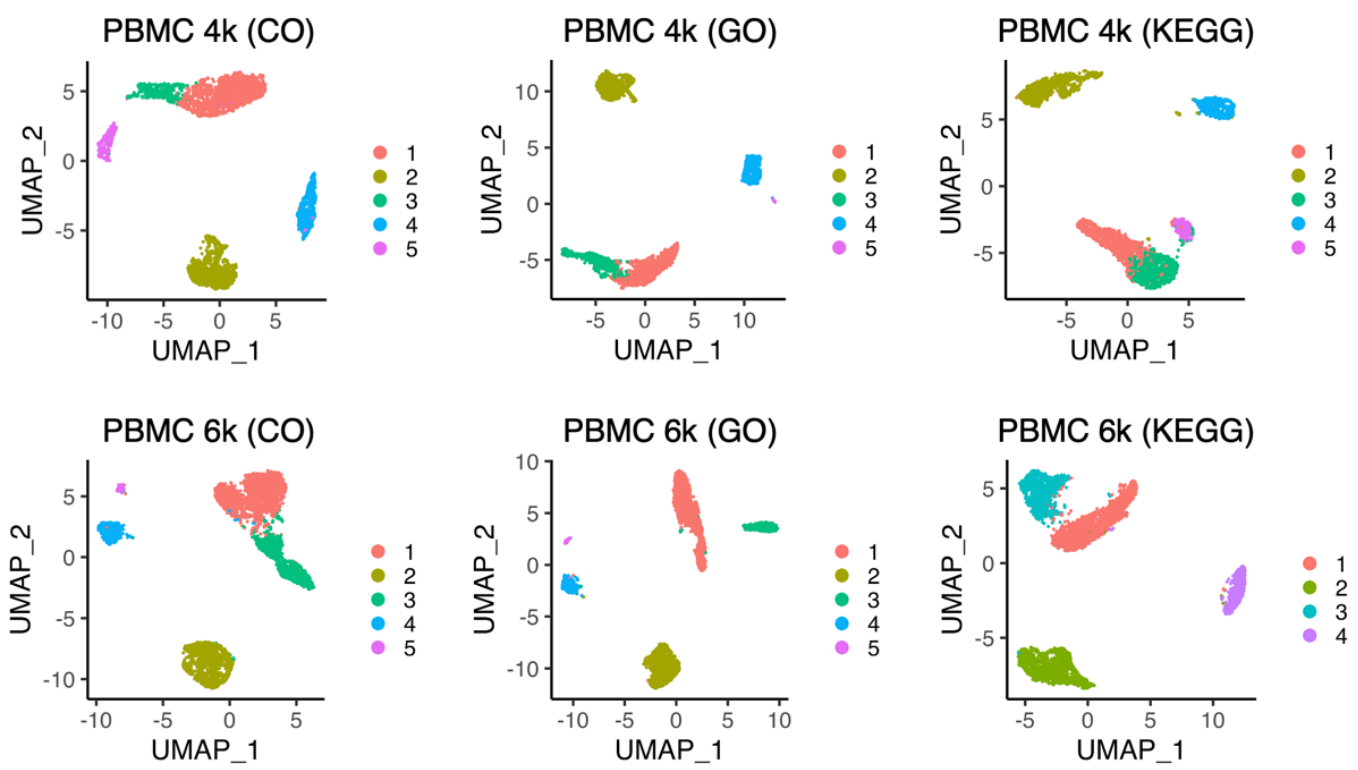

Figure S3. Clustering peripheral blood mononuclear cell (PBMC) 4k and 6k single-cell transcriptomes using ASURAT. Uniform Manifold Approximation and Projection (UMAP) plots of sign-by-sample matrices for Cell Ontology (CO), Gene Ontology (GO), and Kyoto Encyclopedia of Genes and Genomes (KEGG). 


\section{Lung cancer scRNA-seq}

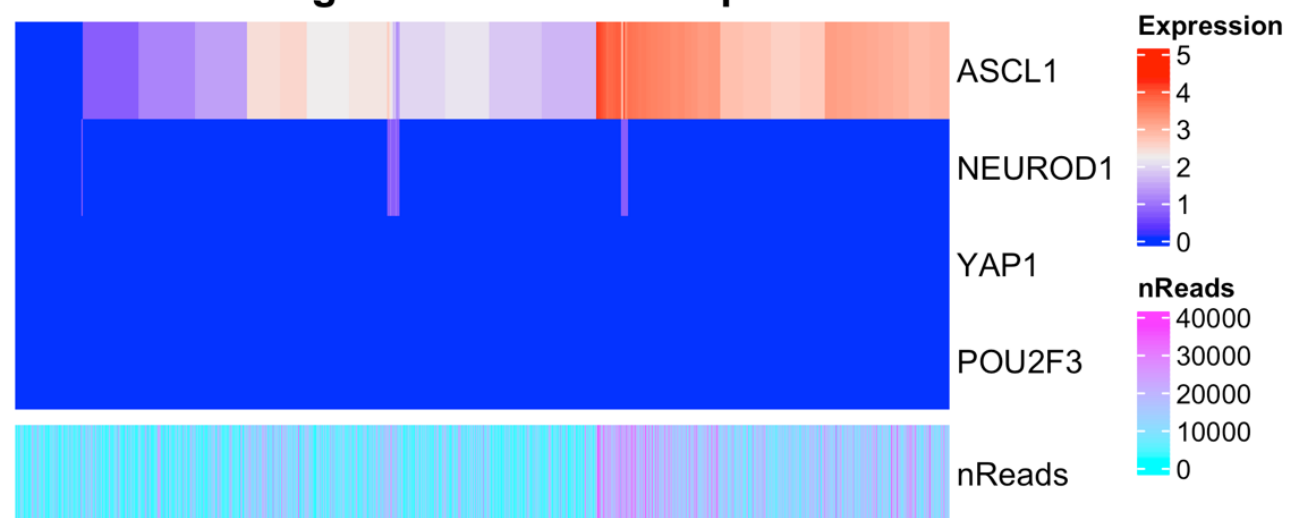

Figure S4. Heatmaps of expression levels of known small cell lung cancer marker genes. Log-normalized gene expression levels for ASCL1, NEUROD1, YAP1, and $P O U 2 F 3$ across all the cells after controlling for data quality. nReads, total read counts. 

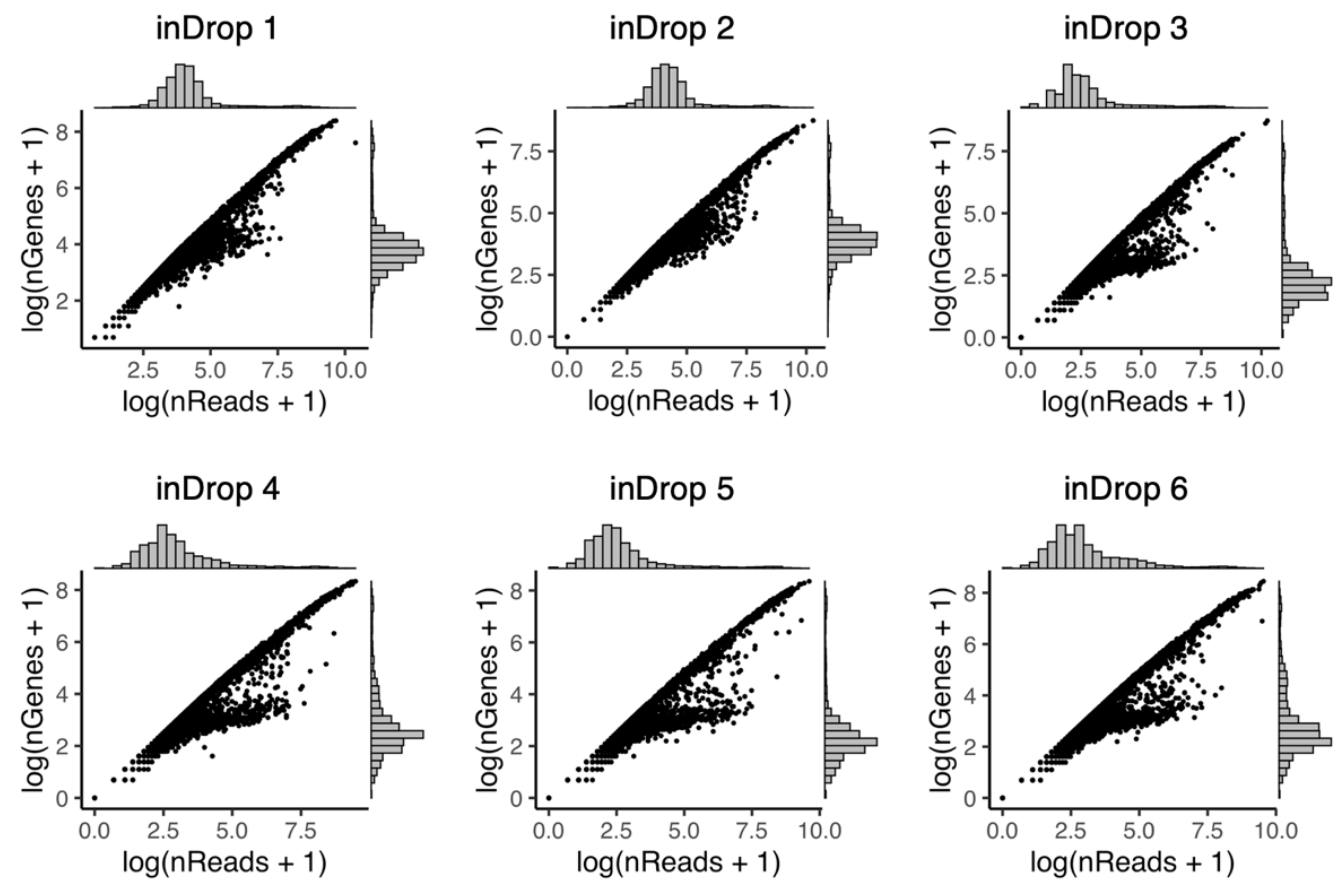

Figure S5. Data qualities across all the cells in single-cell RNA sequencing datasets PDAC-A inDrop from 1 to 6 . nReads, total read counts; nGenes, number of genes expressed with non-zero read counts. 
bioRxiv preprint doi: https://doi.org/10.1101/2021.06.09.447731; this version posted October 12, 2021. The copyright holder for this preprint (which was not certified by peer review) is the author/funder. All rights reserved. No reuse allowed without permission.

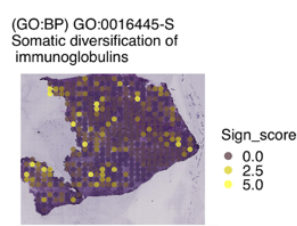

(GO:BP) GO:0044262-S

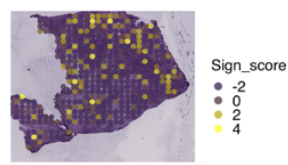

(GO:BP) GO:0051668-S

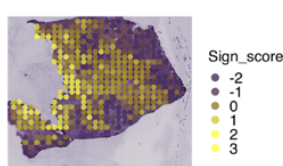

(GO:BP) GO:0051642-S

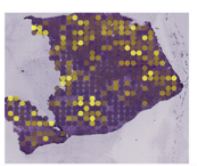

(GO:BP) GO:0001649-S
Osteoblast differentiation

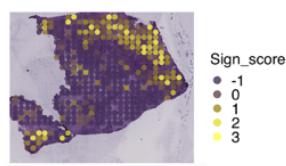

(GO:BP) GO:0040014-S Regulation of multicellular organism

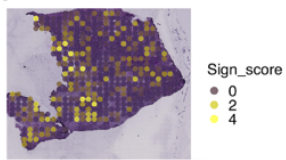

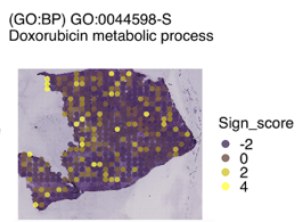

(GO:BP) GO:0044262-V

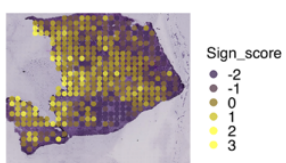

(GO:BP) GO:0098739-S

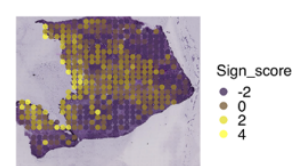

(GO:BP) GO:0061982-S
Meiosis I cell cycle process

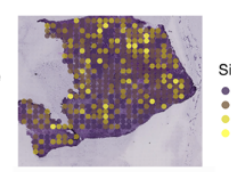

(GO:BP) GO:0001649-V

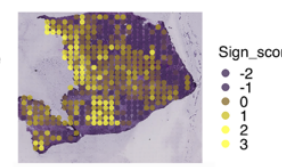

(GO:BP) Anatomical structure maturation

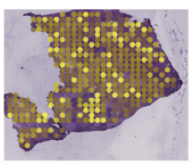

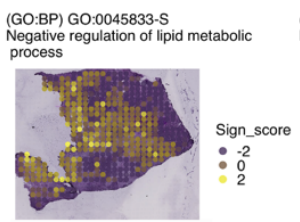

(GO:BP) GO:0006623-S

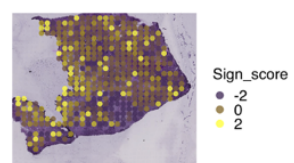

(GO:BP) GO:0098739-V

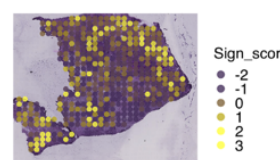

(GO:BP) GO:1903779-S

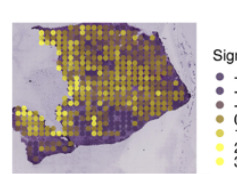

(GO:BP) GO:0050663-S

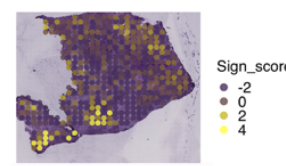

(GO:BP) GO:0071695-V

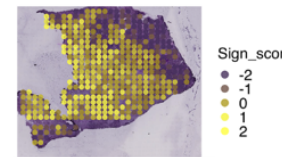

(GO:BP) GO:0120162-S

ositive regutation of cold indued

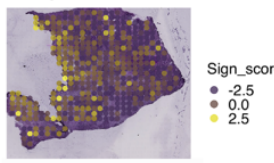

GO:BP) GO:2001021-S

egative regulation of response to DNA

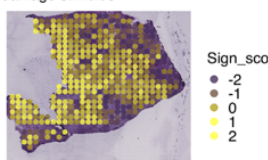

(GO:BP) GO:1903825-S

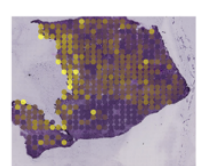

ansport

(GO:BP) GO:1903825-V

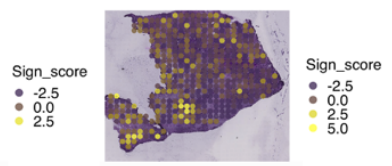

(GO:BP) GO:0050806-S transmissionlation of synaptic (GO:BP) GO:0050806-V
Positive regulation of synapt

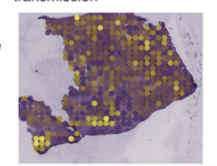

transmission

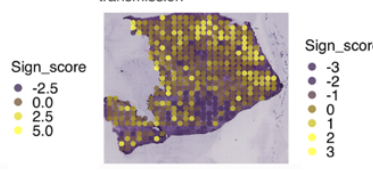

(GO:BP) GO:0007588-S

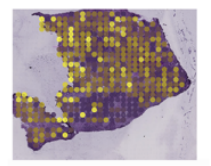

(GO:BP) GO:0009798-S
Axis specification

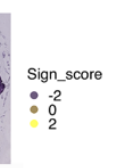

GO:BP) GO:0030902-S

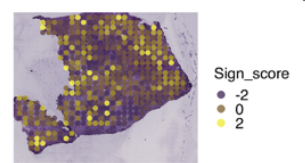

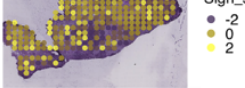

(1) Negative eregulation of NF-kappaB

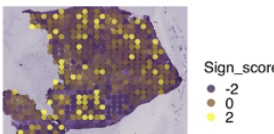


bioRxiv preprint doi: $\mathrm{https}$ //doi.org/10.1101/2021.06.09.447731: this version posted October 12, 2021. The copyright holder for this preprint (which was not certified by peer review) is the author/funder. All rights reserved. No reuse allowed without permission.

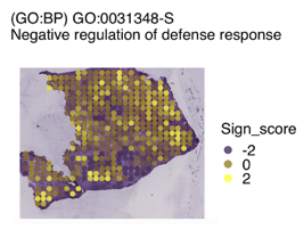

(GO:BP) GO:0002027-S

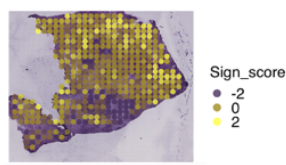

(GO:BP) GO:0002920-V
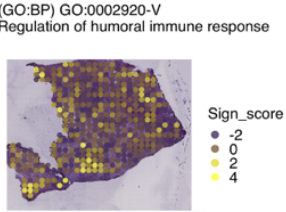

(GO:BP) GO:0002455-V Humoral immune response
circulating immunoglobulin

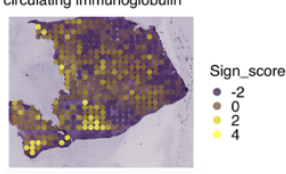

(GO:BP) GO:0044272-S

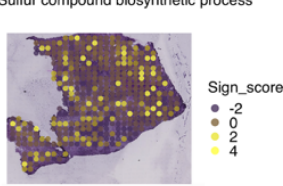

(GO:BP) GO:0051052-S

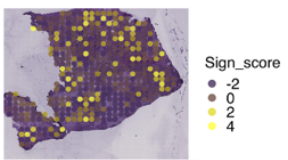

(GO:BP) GO:0031348-V

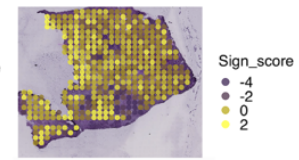

(GO:BP) GO:0008217-S

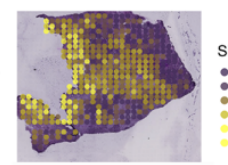

(GO:BP) GO:1902106-S

Negative regulation of leukocyte

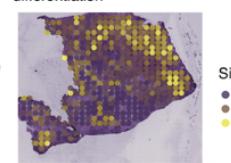

(GO:BP) GO:0034249-S
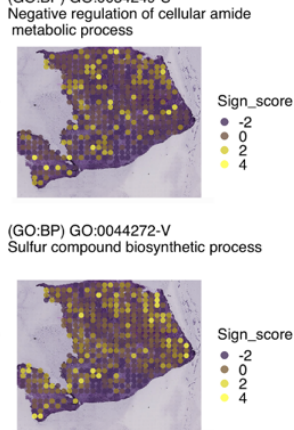

(GO:BP) GO:0051052-V

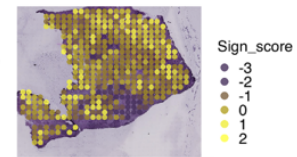

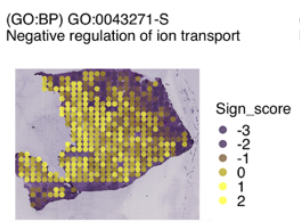

(GO:BP) GO:0010883-S
Regulation of lipid storage

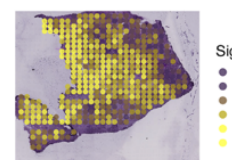

(GO:BP) GO:1902106-V

Negative regulation of leukocyte

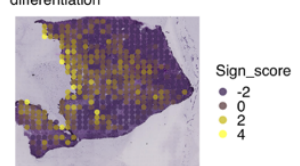

(GO:BP) GO:0046470-S

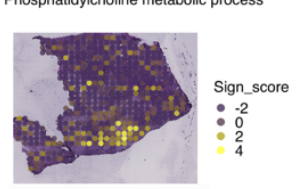

(GO:BP) GO:2000378-S
Negative regulatiton of reactive oxygen
species metabolic process

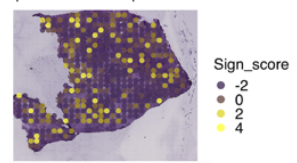

(GO:BP) GO:0006749-S

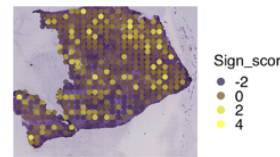

(GO:BP) GO:0009612-S

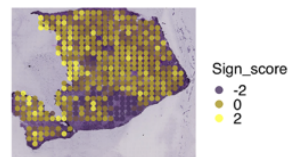

(GO:BP) GO:0007292-S

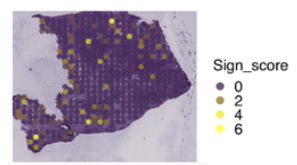

(GO:BP) GO:0002821-S
Positive regulation of adaptive immune response

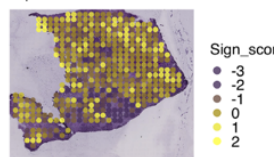

(GO.BP) G0.0046470-V
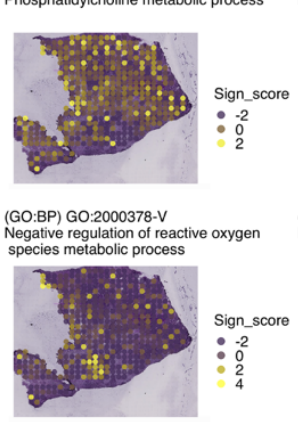

(GO:BP) GO:0006749-V

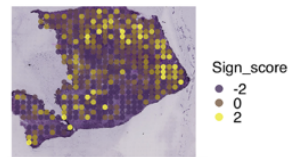

(GO:BP) GO:0009612-V

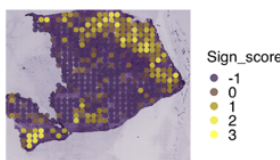

(GO:BP) GO:0002920-S

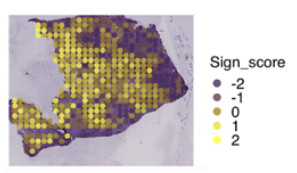

(GO:BP) GO:0002455-S circulat immune response mediated by

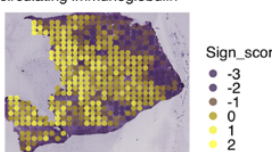

(GO:BP) GO:1903428-S Positive regulation of reactive oxygen
species biosynthetic process

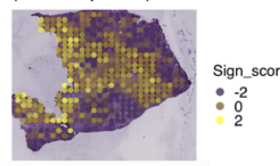

(GO:BP) GO:0002092-S Positive regulation of receptor
internalization

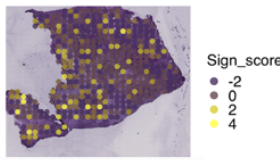

(GO:BP) GO:0042448-S
Progesterone metabolic process

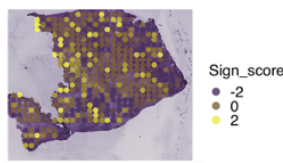


bioRxiv preprint doi: https://doi.org/10.1101/2021.06.09.447731; this version posted October 12, 2021. The copyright holder for this preprint (which was not certified by peer review) is the author/funder. All rights reserved. No reuse allowed without permission.
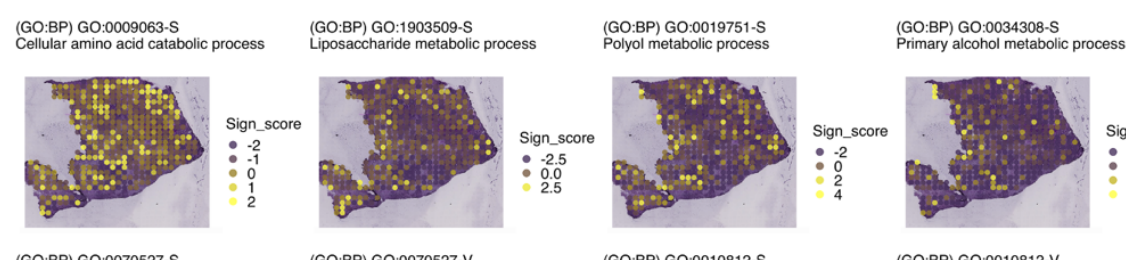

(GO:BP) GO:1901657-S

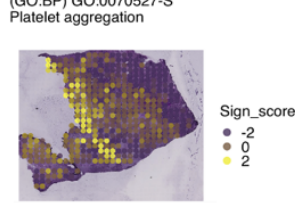

(GO:BP) GO:0070527-V

(GO:BP) GO:0010812-S

(GO:BP) GO:0010812-V

Negative regulation of cell-substrate

Negative re
adhesion
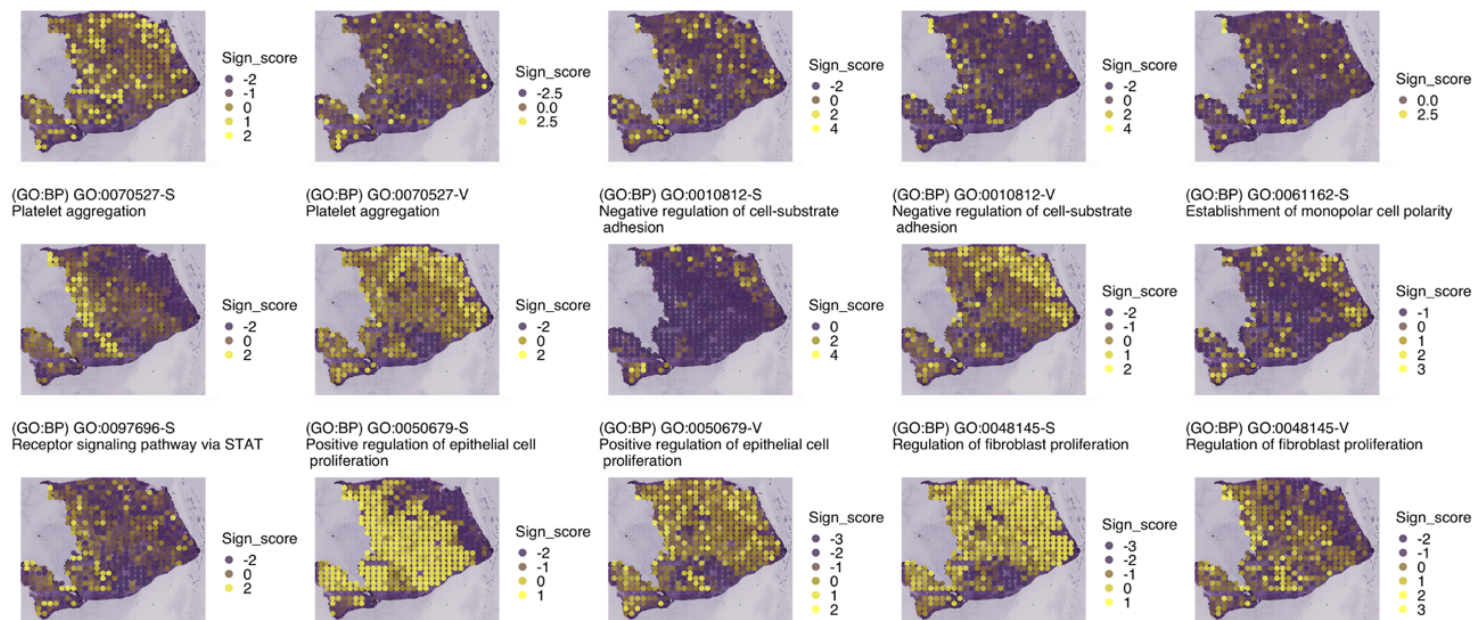

(GO:BP) GO:0050679-S Positive regulation of ep pithelial cell
proliferation

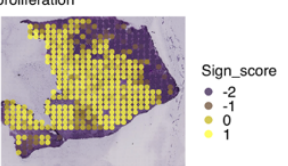

(GO:BP) GO:0050679-V
Positive regulation of epithelial cell

(GO:BP) GO:0048145-S
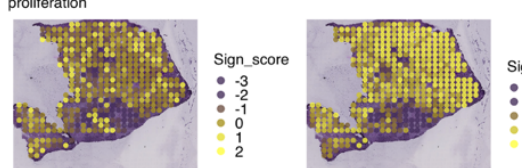

GO:BP) GO:0061162-S
Establishment of monopolar cell polarity

(GO:BP) GO:0043277-S
Apoptotic cell clearance

(GO:BP) GO:0006892-S
Post-Golgi vesicle-mediated

(GO:BP) GO:0140014-S
Mitotic nuclear division

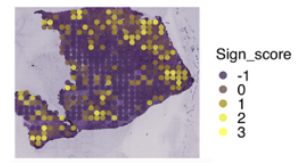

GO:BP) GO:0048145-V
Regulation of fibroblast proliferation
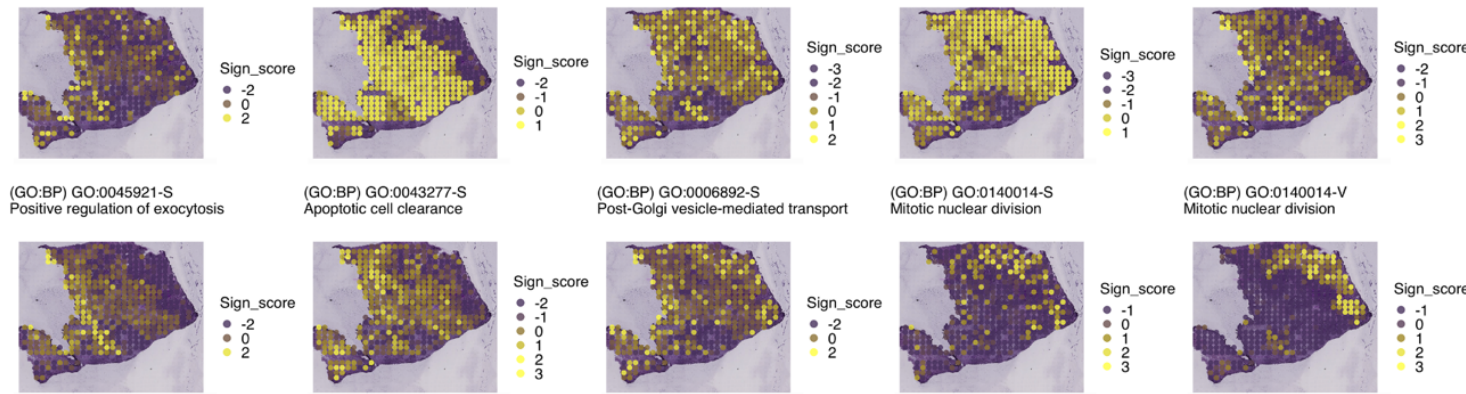

(GO:BP) GO:0031532-S
Actin cytoskeleton reorganization

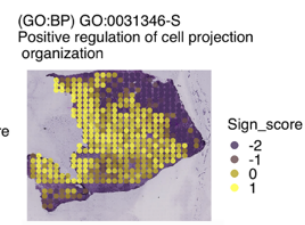

(GO:BP) GO:0031346-V
Positive regulation of cell projection
organization

(GO:BP) GO:0030857-S
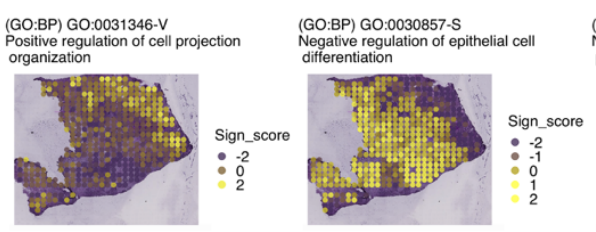

GO:BP) GO:1902904 Negative regulation of supramolecula

(GO:BP) GO:0050709-S

(GO:BP) GO:0050709-V

(GO:BP) GO:0140014-V
Mitotic nuclear division

Negative rogl

protein-containing complex assembly
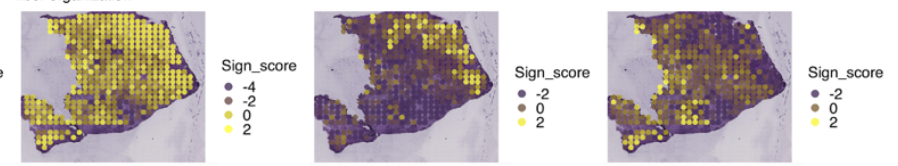

(GO:BP) GO:0021782-S

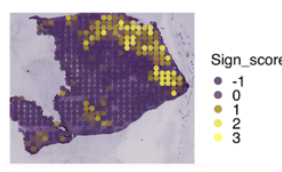

GO:BP) GO:0031333-S

egative regulation of
protein-containing complex assembly
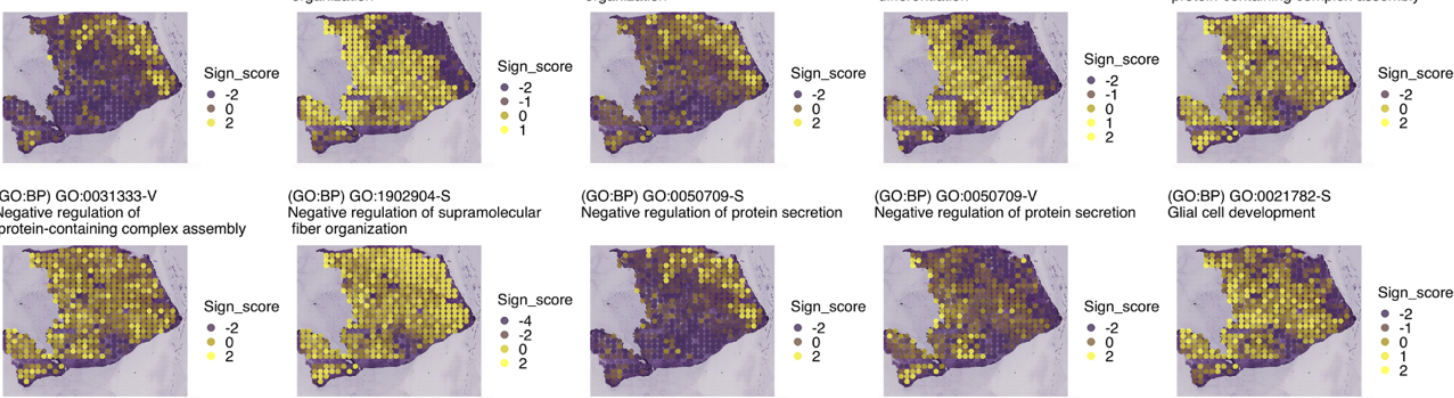
bioRxiv preprint doi: $\mathrm{https}$ //doi.org/10.1101/2021.06.09.447731; this version posted October 12, 2021. The copyright holder for this preprint (which was not certified by peer review) is the author/funder. All rights reserved. No reuse allowed without permission.

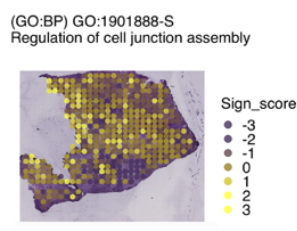

(GO:BP) GO:0032653-S

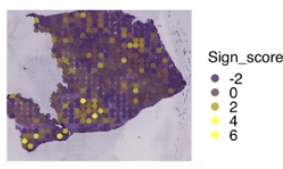

(GO:BP) GO:1903557-S Positive regulation of tumor necrosis
factor superfamily cytokine production
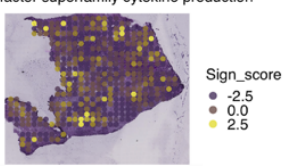

(GO:BP) GO:0001895-V
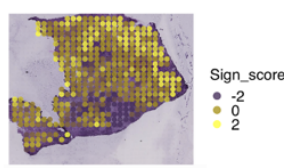

(GO:BP) GO:0090596-S

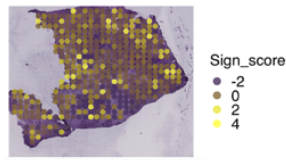

(GO:BP) GO:0016525-V

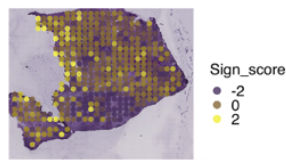

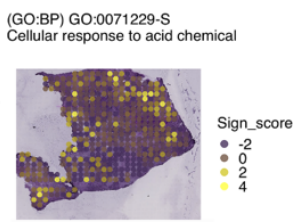

(GO:BP) GO:0032655-S

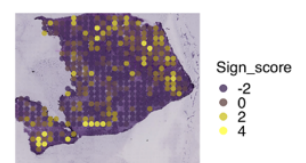

(GO:BP) GO:0021915-S
Neural tube development

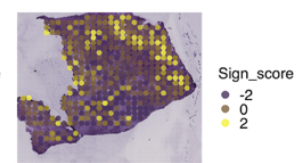

(GO:BP) GO:0010669-S

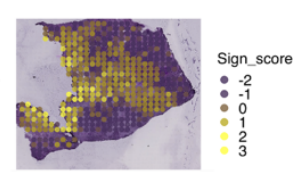

(GO:BP) GO:0035108-S
Limb morphogenesis

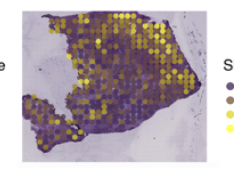

(GO:BP) GO:0030324-S

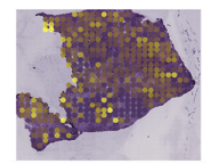

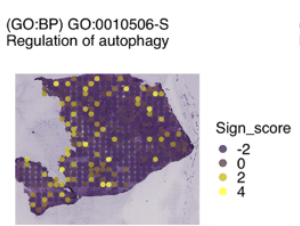

(GO:BP) GO:0032673-S

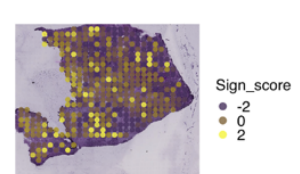

(GO:BP) GO:0055123-S

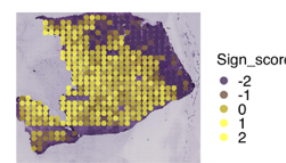

(GO:BP) GO:0045907-S

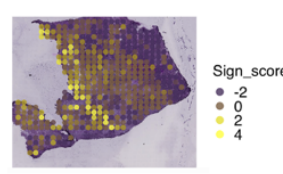

(GO:BP) GO:0060562-S
Epithelial tube morphogenesis

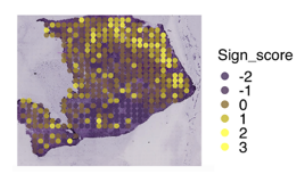

(GO:BP) GO:0044788-S

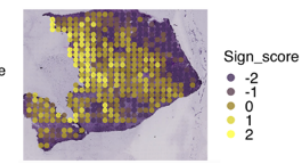

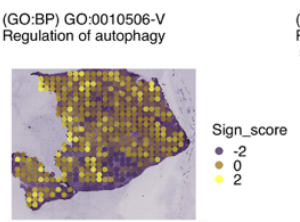

(GO:BP) GO:0071634-S Regulation of transforming growth factor

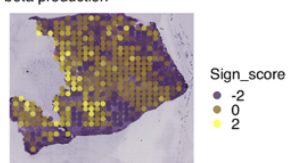

(GO:BP) GO:0009855-S

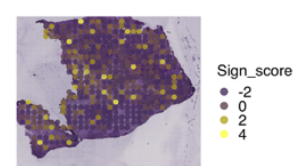

(GO:BP) GO:0022612-S
Gland morphogenesis

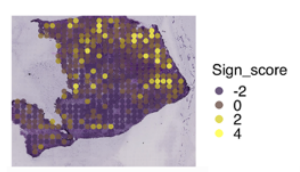

(GO:BP) GO:0060562-V

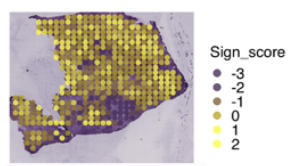

(GO:BP) GO:0061844-S Antimicrobial humoral immune respon
mediated by antimicrobial peptide

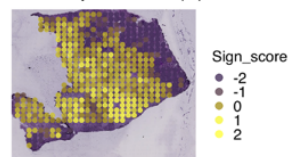

(GO:BP) GO:0090277-S Positive regulation of peptide hormone
secretion

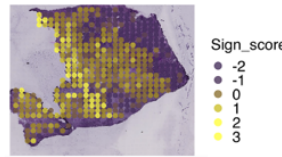

(GO:BP) GO:0032755-S

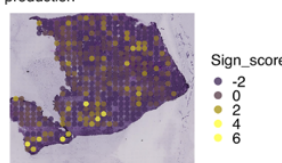

(GO:BP) GO:0001895-S
Retina homeostasis

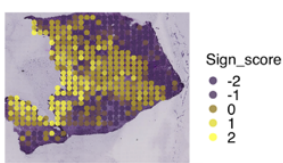

(GO:BP) GO:0048562-S

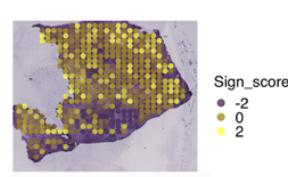

(GO:BP) GO:0016525-S

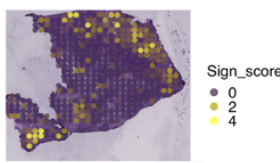

GO:BP) GO:0090303-S

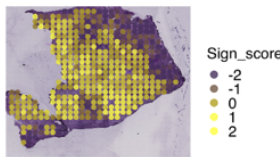


bioRxiv preprint doi: https://doi.org/10.1101/2021.06.09.447731; this version posted October 12, 2021. The copyright holder for this preprint (which was not certified by peer review) is the author/funder. All rights reserved. No reuse allowed without permission.

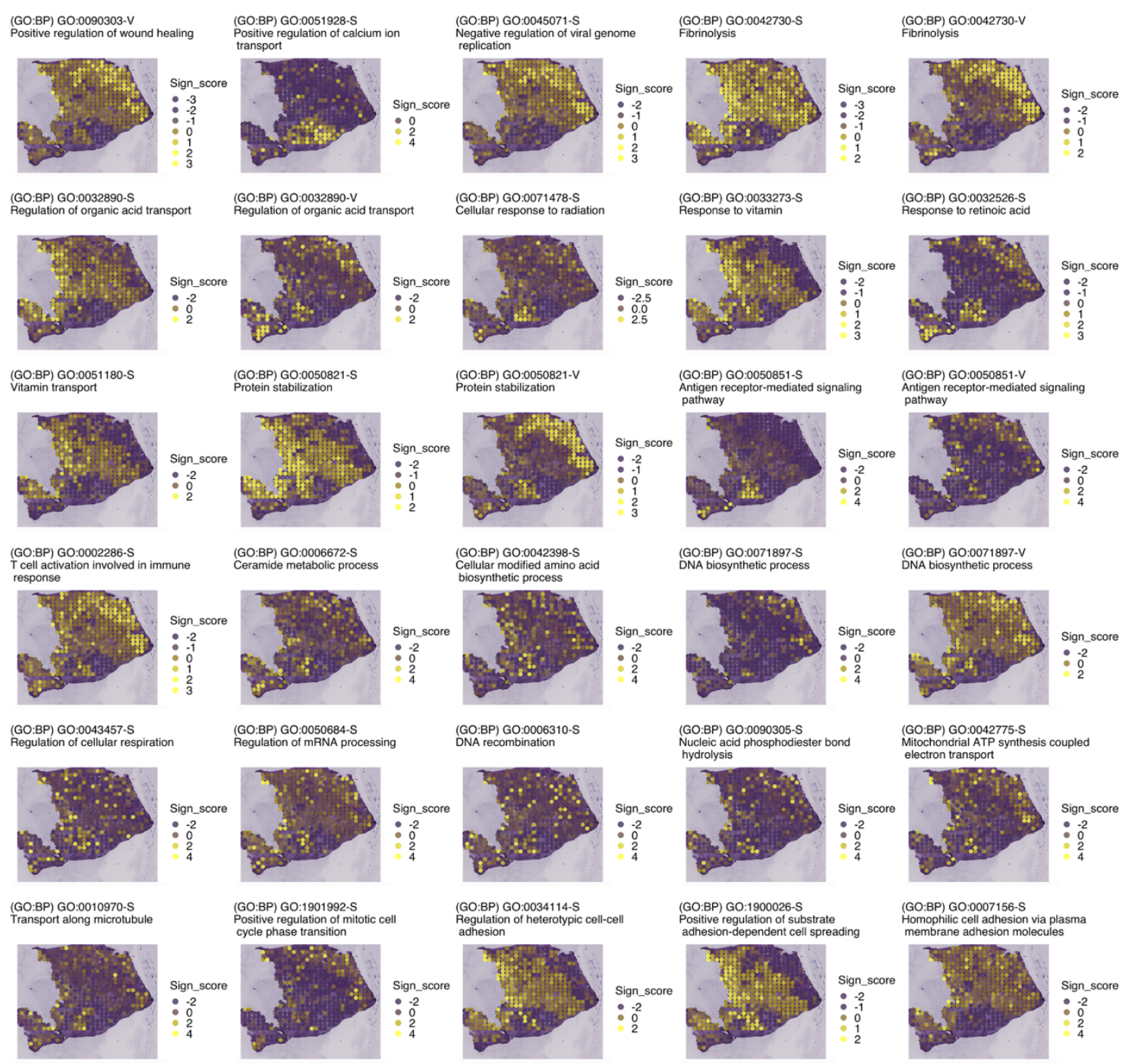


bioRxiv preprint doi: $\mathrm{https}$ //doi.org/10.1101/2021.06.09.447731: this version posted October 12, 2021. The copyright holder for this preprint (which was not certified by peer review) is the author/funder. All rights reserved. No reuse allowed without permission.

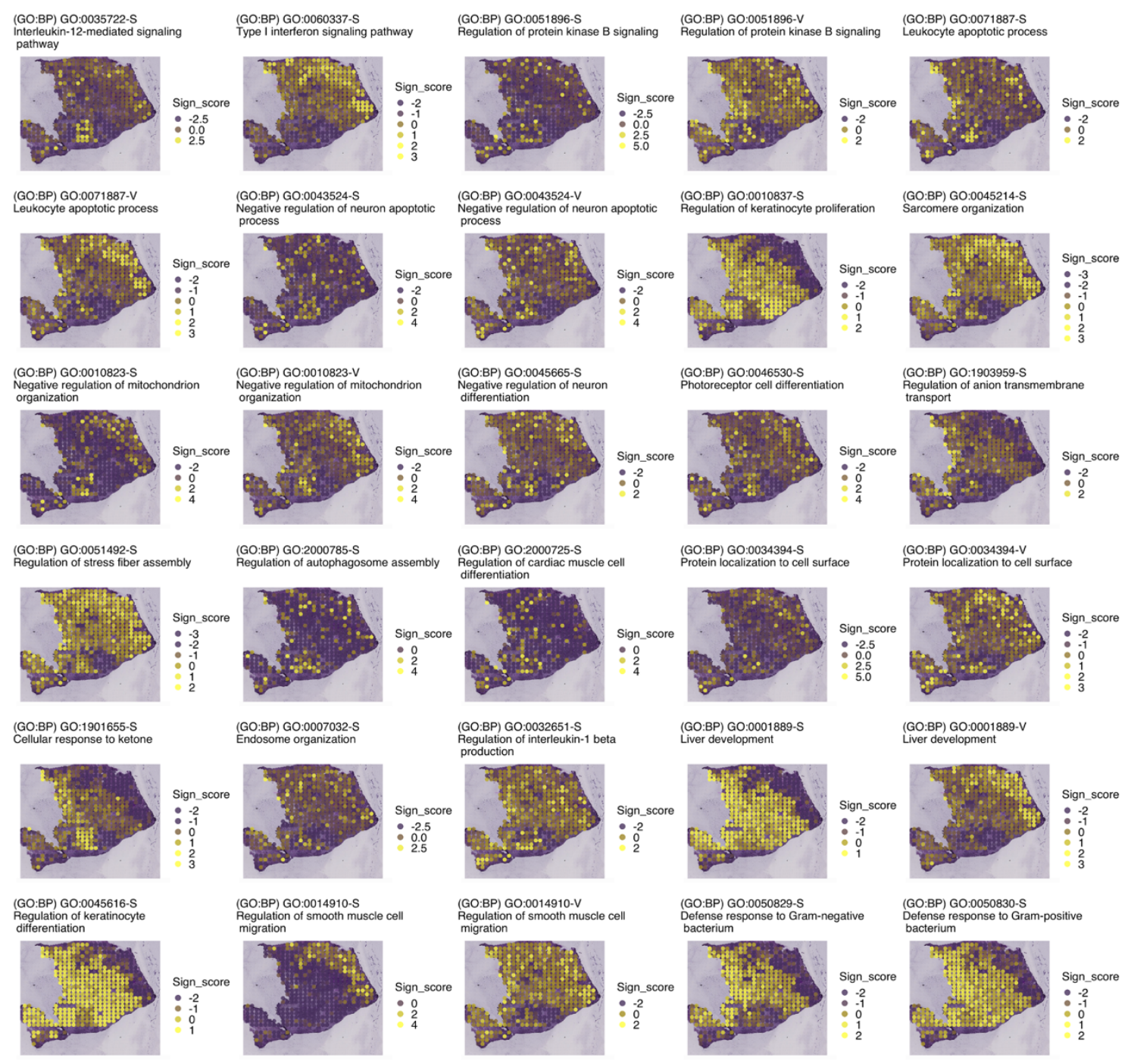


bioRxiv preprint doi: https://doi.org/10.1101/2021.06.09.447731: this version posted October 12, 2021. The copyright holder for this preprint (which was not certified by peer review) is the author/funder. All rights reserved. No reuse allowed without permission.

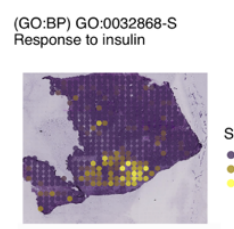

(GO:BP) GO:0098781-V

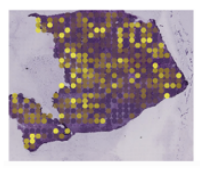

GO:BP) GO:0018279-S (GO:BP) GO:0018279-S
Protein N-linked glycosylation via
asparagine

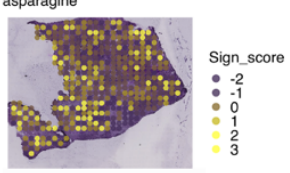

(GO:BP) GO:1902041-S Regulation of extrinsic apoptotic
signaling pathway via death doma

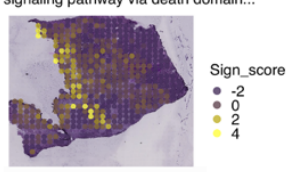

(GO:BP) GO:0010660-S Regulation of muscle cell apoptotic
process

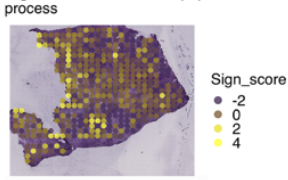

(GO:BP) GO:0036010-S

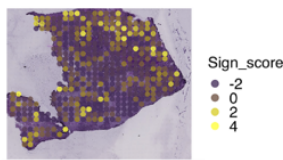

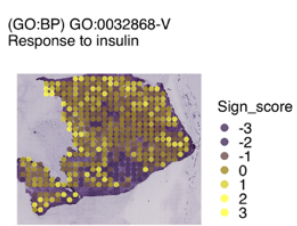

(GO:BP) GO:0019370-S

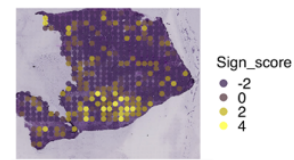

(GO:BP) GO:0016266-S
O-glycan processing

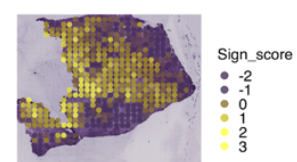

(GO:BP) GO:1902041-V (GO:BP) GO:1902041-V
Regulation of extrinsic apoptotic
signaling pathway via death domain

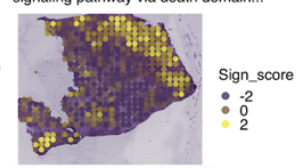

(GO:BP) GO:0043280-S Positive regulation of cysteine-type
endopeptidase activity involved in.

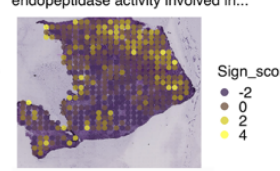

(GO:BP) GO:0071277-S

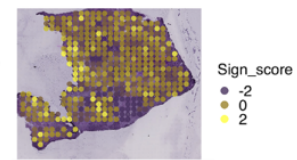

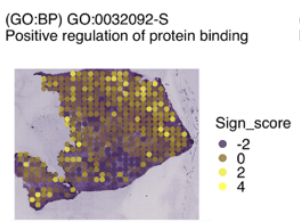

(GO:BP) GO:0032928-S gulation of superoxide anio

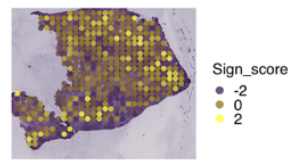

(GO:BP) GO:0006693-S

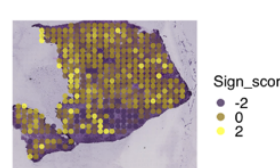

(GO:BP) GO-0046578-S Regulation of Ras protein signal

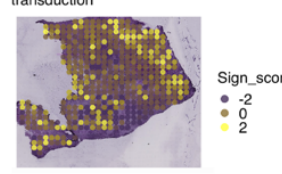

(GO:BP) GO:0030316-S
Osteoclast differentiation

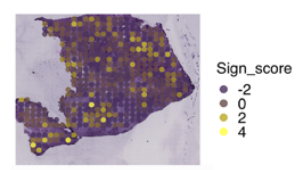

(GO:BP) GO:0071280-S

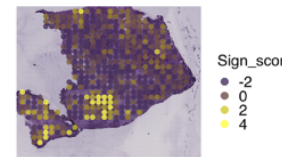

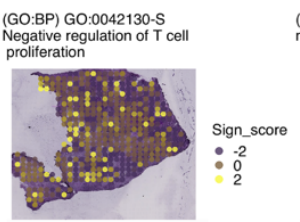

(GO:BP) GO:0098781-S
ncRNA transcription

(GO:BP) GO:0032928-V Regulation of superoxide anio

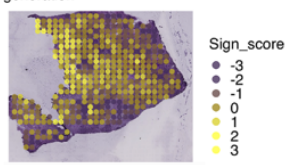
(GO:BP) GO:0010389-S
Regulation of G2/M transition of mitotic

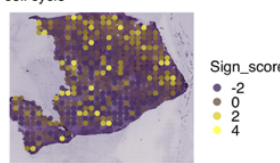
(GO:BP) GO:0070374-S
Positive regulation of ERK1 and ERK2

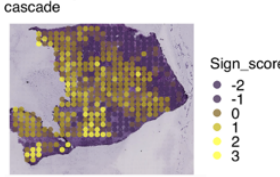

(GO:BP) GO:0030316-V

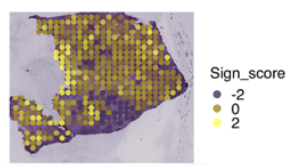

(GO:BP) GO:0071294-S

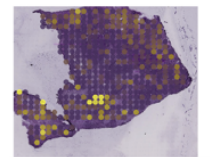

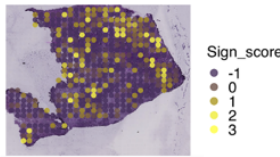

(GO:BP) GO:0043543-S

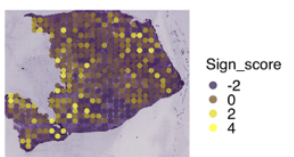

(GO:BP) GO:0048008-S signaling pathwayth factor recept

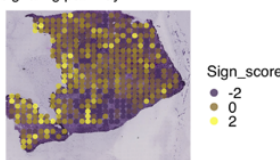

(GO:BP) GO:0070374-V cositive regulation of ERK1 and ERK2
cade

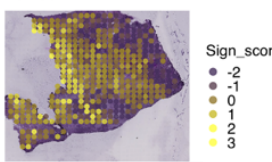

(GO:BP) GO:0034067-S

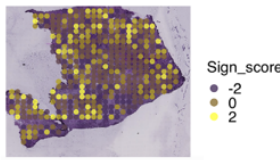

(GO:BP) GO:0071347-S

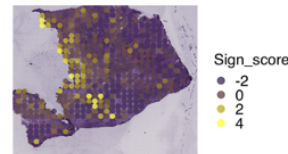


bioRxiv preprint doi: https://doi.org/10.1101/2021.06.09.447731; this version posted October 12, 2021. The copyright holder for this preprint (which was not certified by peer review) is the author/funder. All rights reserved. No reuse allowed without permission.

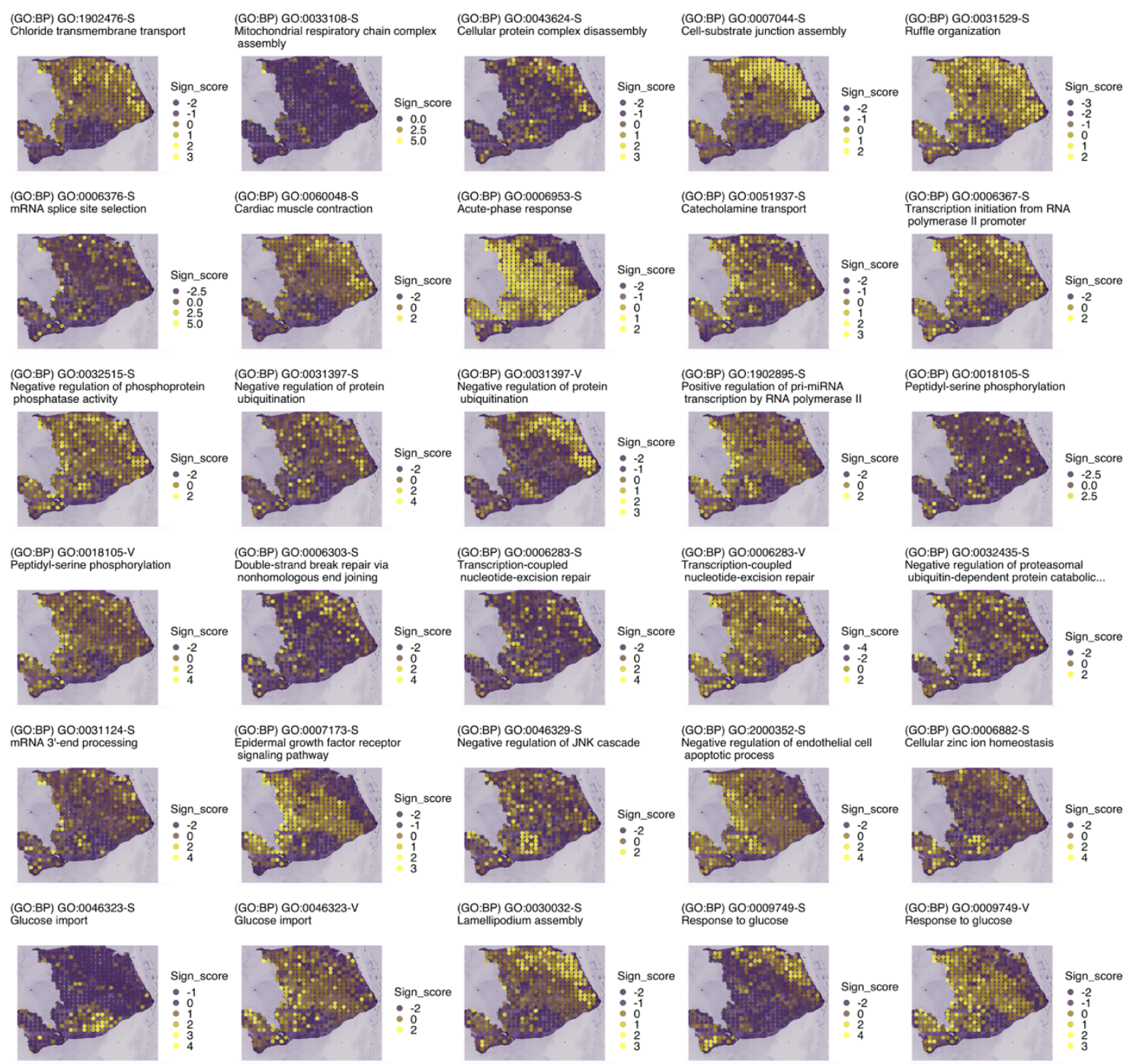


bioRxiv preprint doi: $\mathrm{https}$ //doi.org/10.1101/2021.06.09.447731: this version posted October 12, 2021. The copyright holder for this preprint (which was not certified by peer review) is the author/funder. All rights reserved. No reuse allowed without permission.

(GO:BP) GO:0006813-S
Potassium ion transport

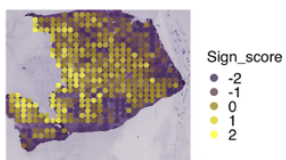

(GO:BP) GO:0055078-S
(GO:BP) GO:0061098-S Positive regulation of protein tyrosine
kinase activity

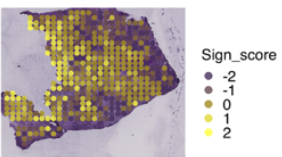

(GO-BP) GO:0000413-S

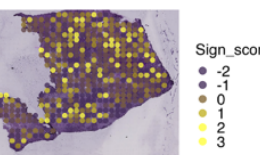

(GO:BP) GO:0000377-S reactions with bulged adenosine as.

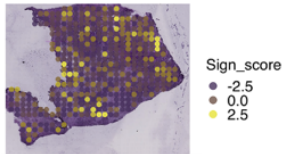

GO:BP) GO.0000377-V

NA splicing, via transesterification

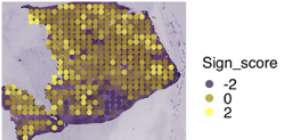

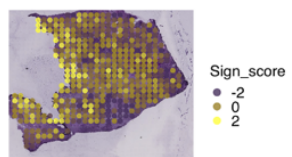


bioRxiv preprint doi: https://doi.org/10.1101/2021.06.09.447731; this version posted October 12, 2021. The copyright holder for this preprint (which was not certified by peer review) is the author/funder. All rights reserved. No reuse allowed without permission.

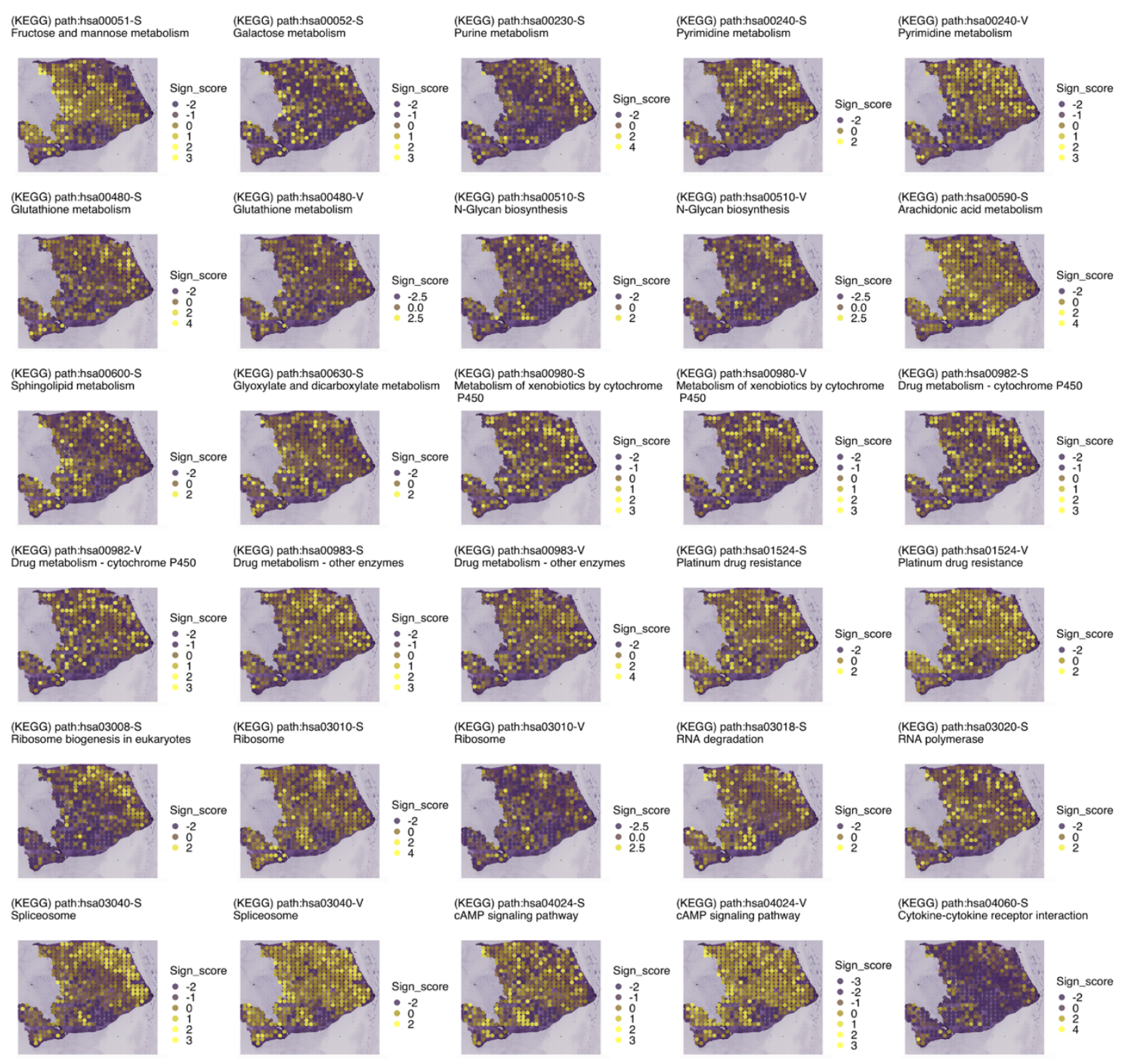


bioRxiv preprint doi: https://doi.org/10.1101/2021.06.09.447731; this version posted October 12, 2021. The copyright holder for this preprint (which was not certified by peer review) is the author/funder. All rights reserved. No reuse allowed without permission.

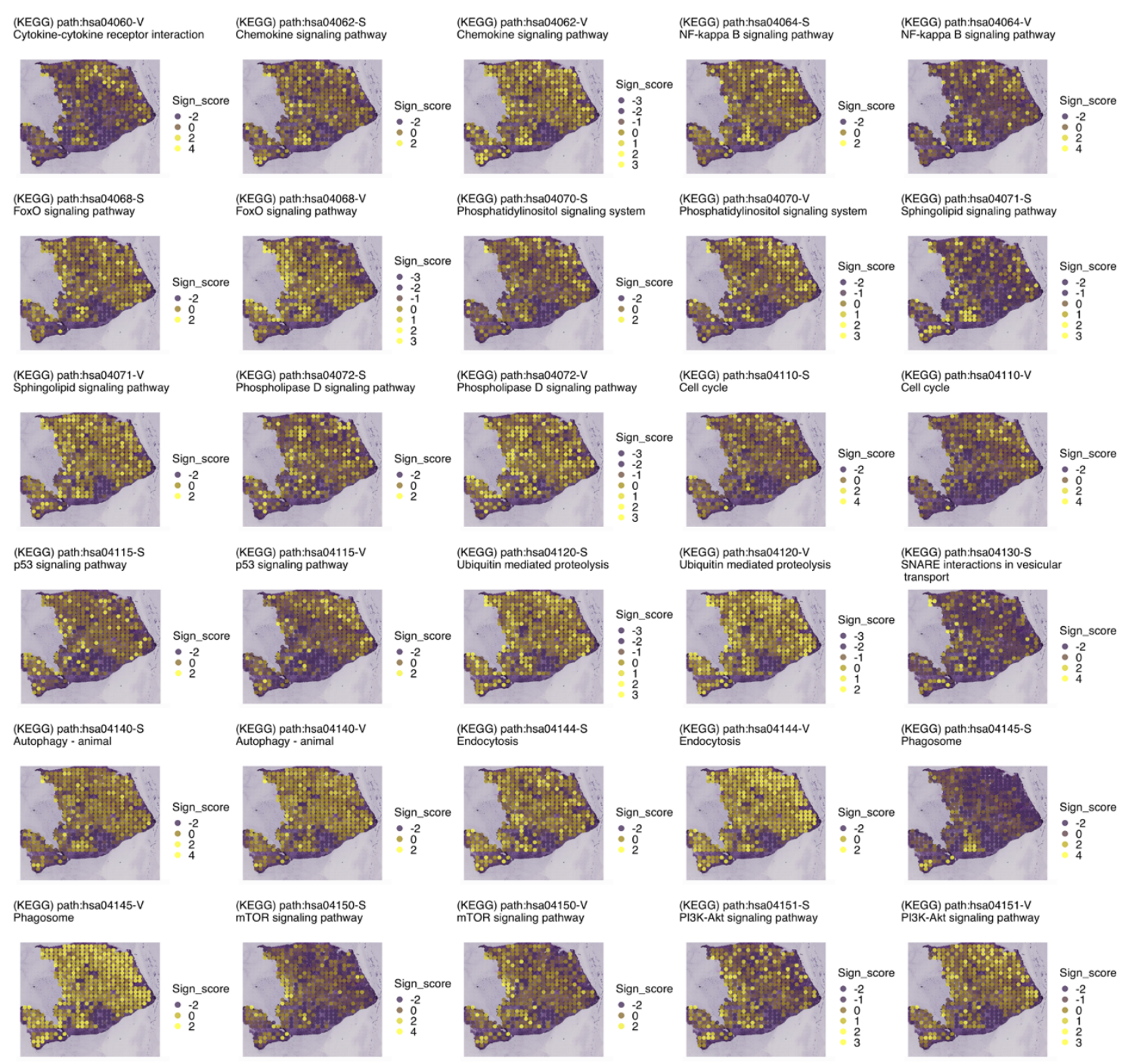


bioRxiv preprint doi: https://doi.org/10.1101/2021.06.09.447731; this version posted October 12, 2021. The copyright holder for this preprint (which was not certified by peer review) is the author/funder. All rights reserved. No reuse allowed without permission.

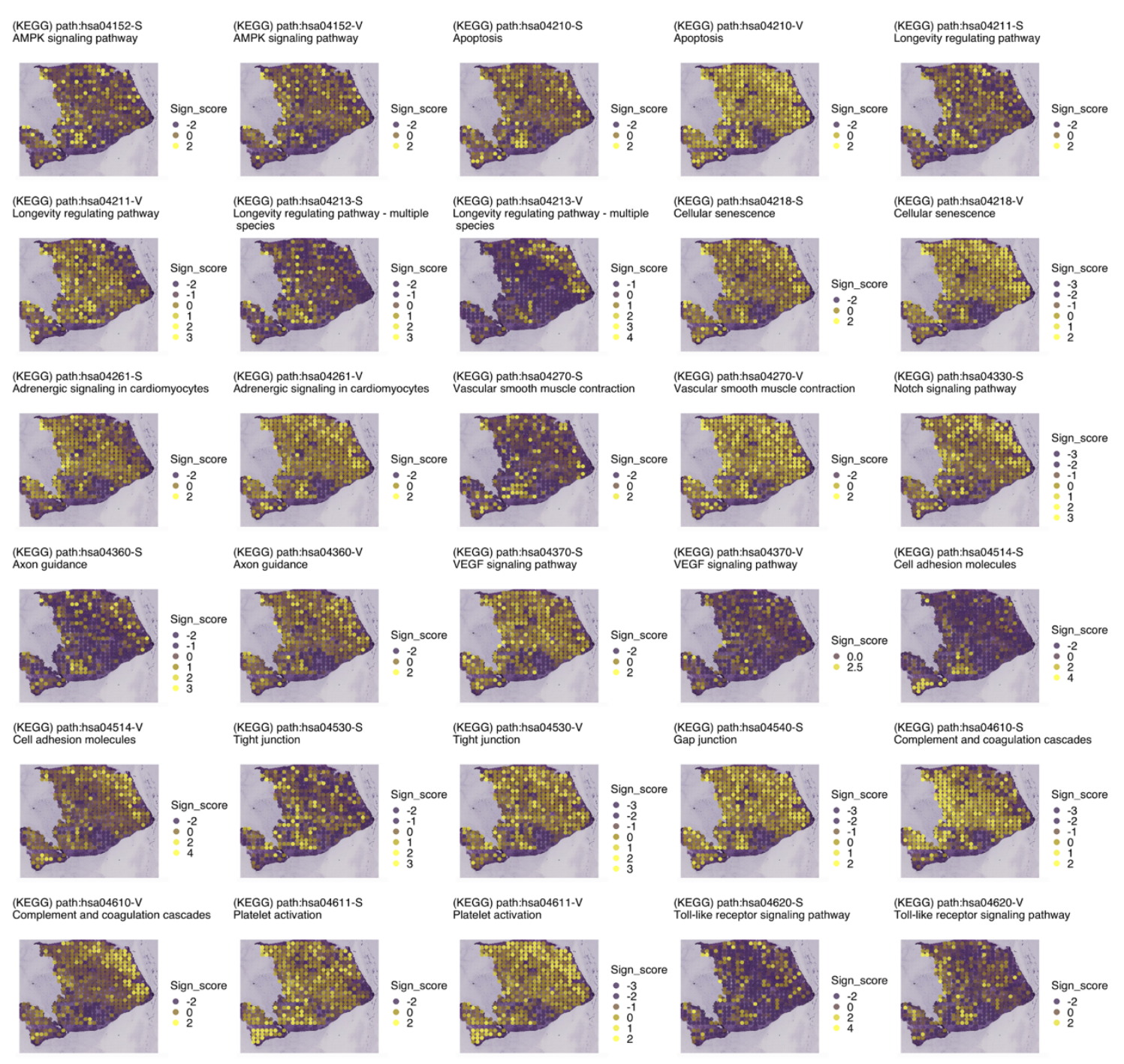


bioRxiv preprint doi: https://doi.org/10.1101/2021.06.09.447731; this version posted October 12, 2021. The copyright holder for this preprint (which was not certified by peer review) is the author/funder. All rights reserved. No reuse allowed without permission.

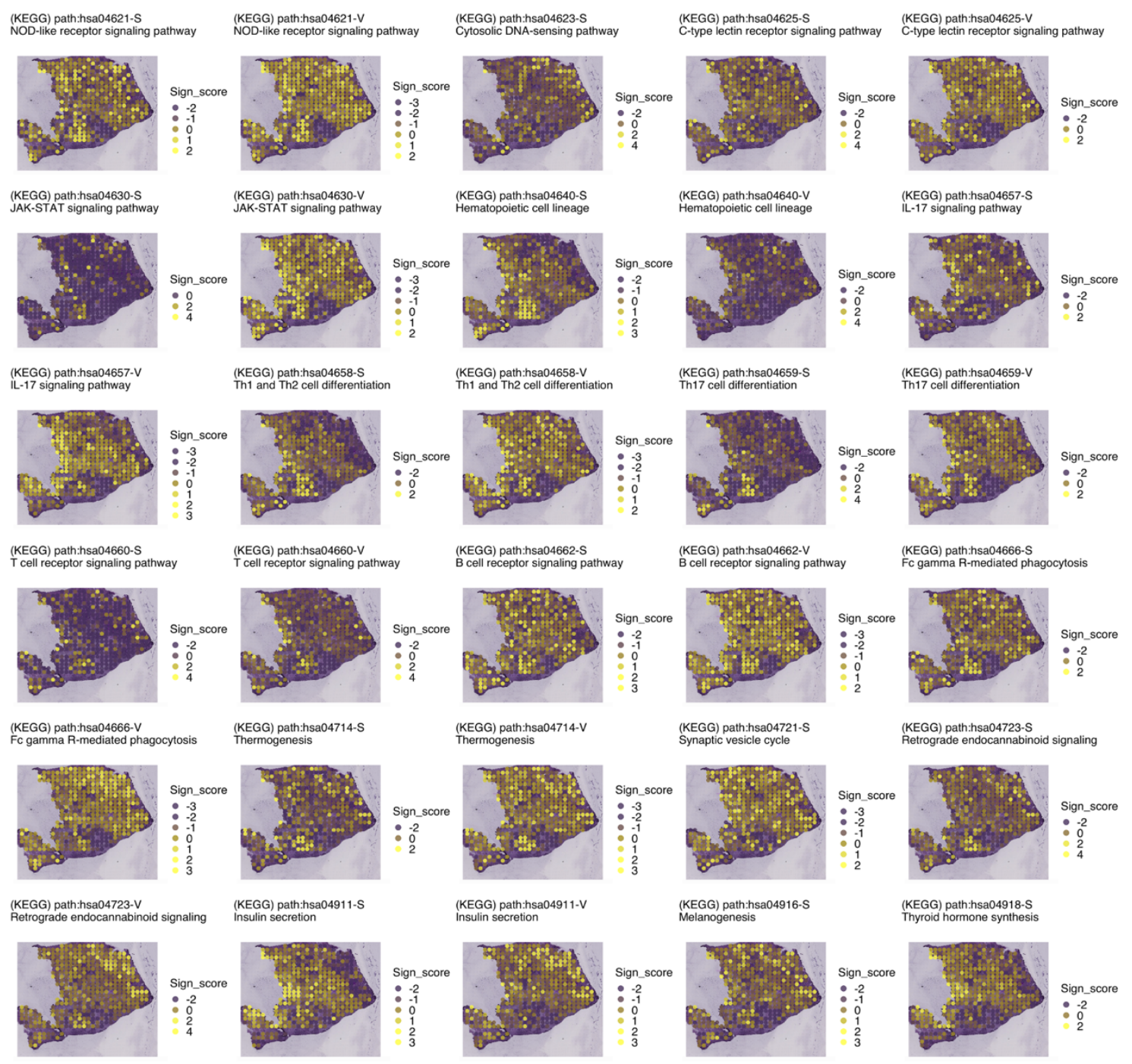


bioRxiv preprint doi: https://doi.org/10.1101/2021.06.09.447731; this version posted October 12, 2021. The copyright holder for this preprint (which was not certified by peer review) is the author/funder. All rights reserved. No reuse allowed without permission.

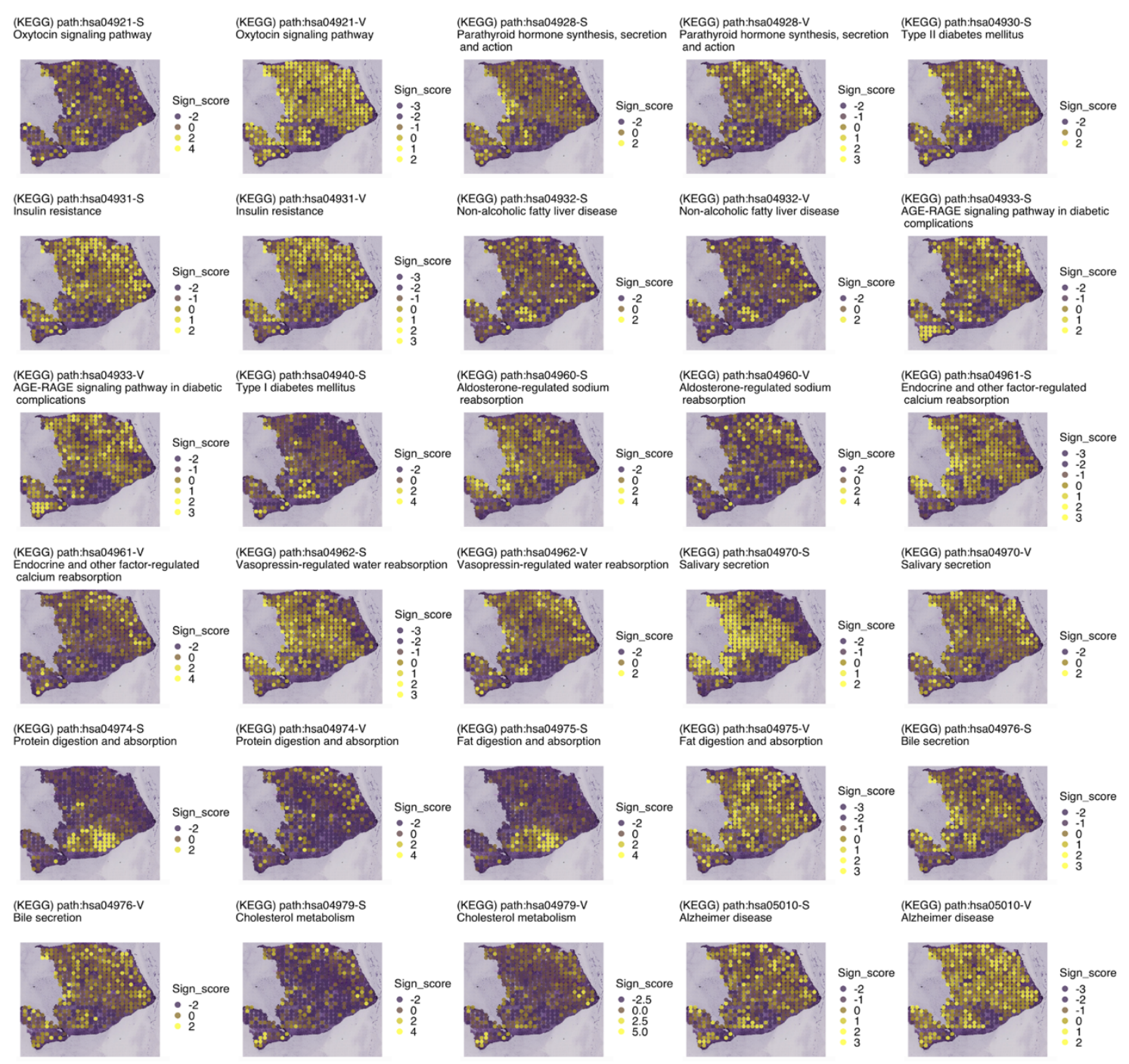


bioRxiv preprint doi: https://doi.org/10.1101/2021.06.09.447731; this version posted October 12, 2021. The copyright holder for this preprint (which was not certified by peer review) is the author/funder. All rights reserved. No reuse allowed without permission.

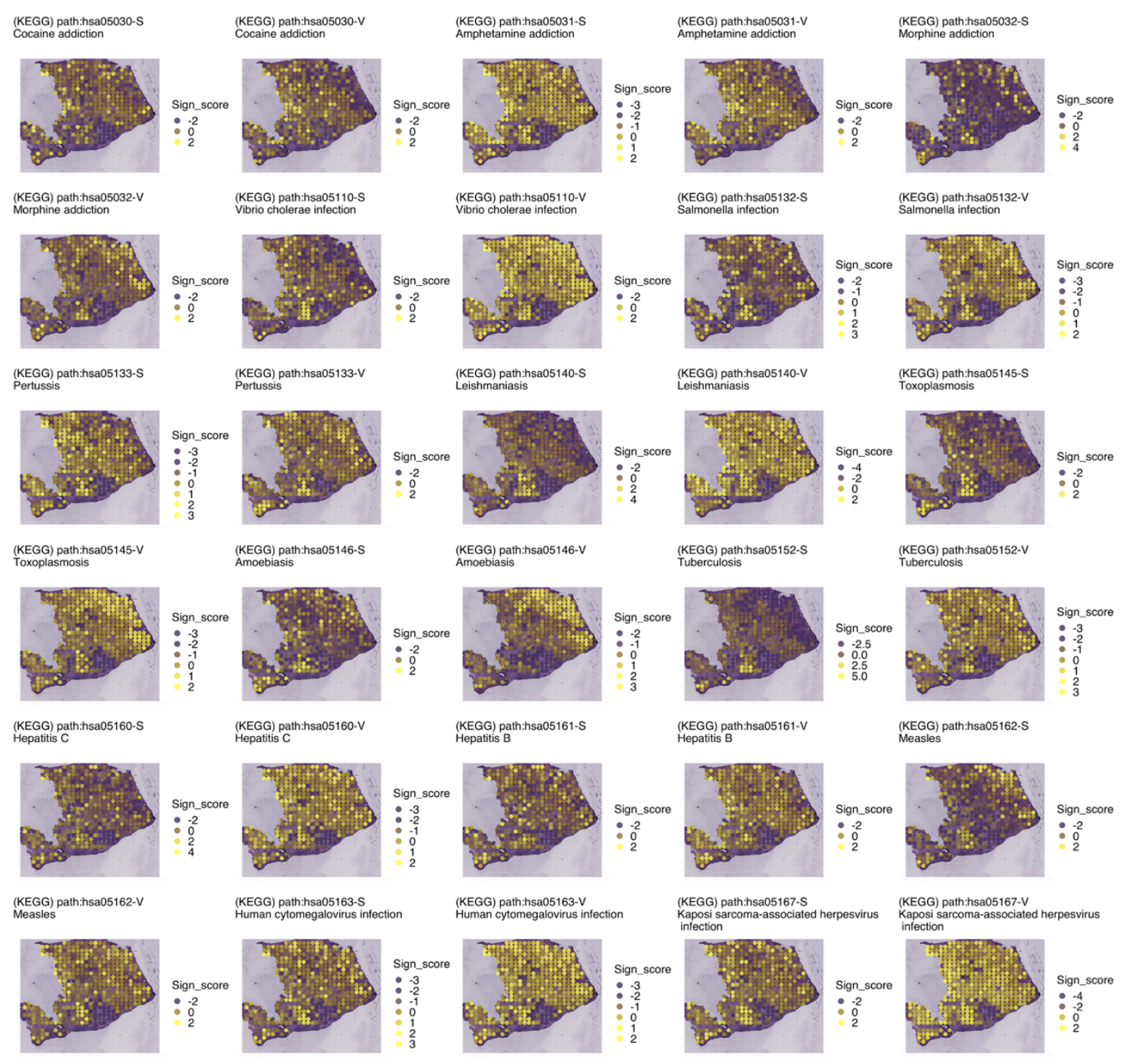




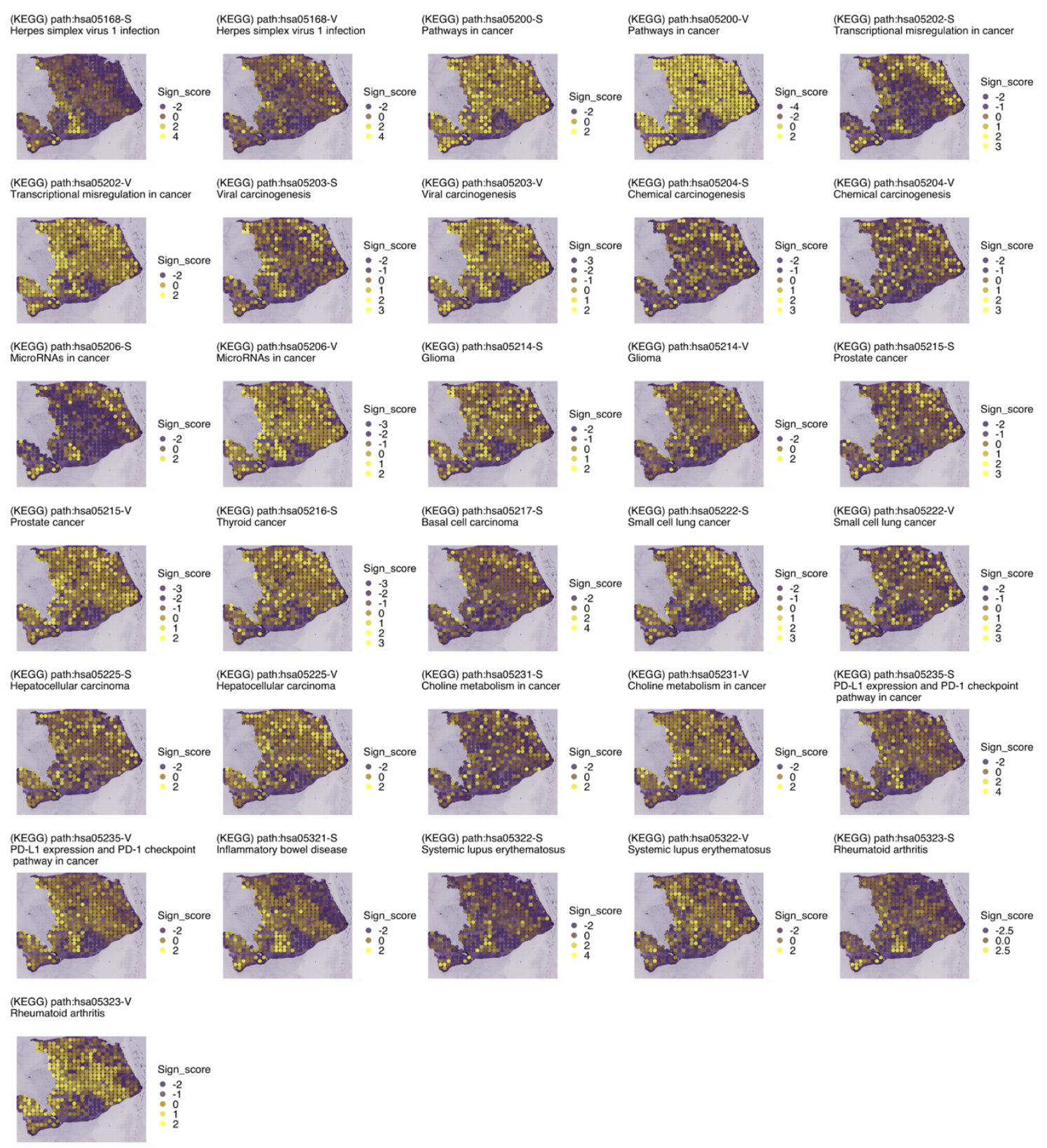

Figure S6. Sign scores for functions and signaling pathway activities using Gene Ontology (GO) and Kyoto Encyclopedia of Genes and Genomes (KEGG) across the pancreatic ductal adenocarcinoma (PDAC) tissue. 


\section{Supplementary Note 1. Parameter settings of ASURAT}

To obtain desired results, it is critical to tune ASURAT parameters for creating sign-bysample matrices (SSMs). Depending on the DBs, there were six to nine parameters for creating SSMs, but many of them have been preset to unbiased and sensible default values (Figure S1). We found that our default settings worked well in our single-cell RNA sequencing (scRNA-seq) analyses, but the three parameters should be tuned by users, as described below.

As formulated in Error! Reference source not found., positive and negative constants $\alpha$ and $\beta$ from thresholds of correlation coefficients are required for decomposing correlation graphs and creating signs (see Figure 2 for the demonstration). In addition, unreliable signs are discarded with user-defined criteria, which were preset as follows: the sum of the number of genes in the strongly and variably correlated gene sets, SCG and VCG, respectively, is less than $n_{\min }$ or the number of genes in weakly correlated gene set (WCG) is less than $n_{\mathrm{min}}^{(\mathrm{w})}$ (the default value is 2). Furthermore, users can remove redundant signs with similar biological meanings if information contents (ICs) (Yu, et al., 2010) are defined.

\section{Supplementary Note 2. Separation index}

Briefly, a separation index is a measure of significance of a given sign score for a given subpopulation. Since the row vectors of SSMs are centered (i.e., the means are zeros), wherein the degree of freedom is reduced, naïve usages of statistical tests and fold change analyses should be avoided. Nevertheless, we propose helping users to find significant signs using a nonparametric index to quantify the extent of separation between two sets of random variables. A separation index of a given random variable $X$ takes a value from -1 to 1: the larger positive value indicates that $X \mathrm{~s}$ are markedly upregulated, and the probability distribution is well separated against other distributions and vice versa.

Let us consider a vector $\boldsymbol{a}$ of size $n$, i.e., the number of samples, whose elements stand for the sign scores, and assume that the elements are sorted in ascending order. For simplicity suppose that the samples are classified into two clusters labeled 0 and 1 . Let $\boldsymbol{v}$ be a vector of the labels corresponding to $\boldsymbol{a}$, and $\boldsymbol{w}_{0}$ and $\boldsymbol{w}_{1}$ be vectors having the same 
elements with $\boldsymbol{v}$ but the elements are sorted in lexicographic orders in forward and backward directions, respectively. Then, we define the separation index as follows:

$$
I(\boldsymbol{v})=1-\frac{2 d\left(\boldsymbol{v}, \boldsymbol{w}_{0}\right)}{d\left(\boldsymbol{v}, \boldsymbol{w}_{0}\right)+d\left(\boldsymbol{v}, \boldsymbol{w}_{1}\right)}
$$

where $d\left(\boldsymbol{v}, \boldsymbol{w}_{i}\right)$ is an edit distance (or Levenshtein distance (Lowrance and Wagner, 1975)) with only adjacent swapping permitted. For example, if $\boldsymbol{v}=(1,0,0,1,1)$, then $\boldsymbol{w}_{0}=(0,0,1,1,1)$ and $\boldsymbol{w}_{1}=(1,1,1,0,0)$. From (3) one can calculate $d\left(\boldsymbol{v}, \boldsymbol{w}_{0}\right)=2$ and $d\left(\boldsymbol{v}, \boldsymbol{w}_{1}\right)=4$, and thus $I(\boldsymbol{v})=1 / 3$. As another example, if $\boldsymbol{v}=(0,1,1,0,0)$, then $I(\boldsymbol{v})=-1 / 3$. From this example, one can see that the positive and negative values of $I$ mean that the given sign has positive and negative contributions for cluster " 1 ," respectively.

\section{Supplementary Note 3. Datasets}

Human peripheral blood mononuclear cells

These data were obtained from peripheral blood mononuclear cells (PBMCs) of healthy donors, which include approximately 4,000 and 6,000 cells; thus, they were referred to as PBMCs $4 \mathrm{k}$ and $6 \mathrm{k}$, respectively. The data were produced with a 10x protocol using unique molecular identifiers (UMIs). The single-cell transcriptome datasets were downloaded from the 10x Genomics repository (https://support.10xgenomics.com/single-cell-geneexpression/datasets). The following filtered read count matrices were obtained: PBMC $4 \mathrm{k}$ from a healthy donor (https://support.10xgenomics.com/single-cell-geneexpression/datasets/2.1.0/pbmc4k) and PBMC $6 \mathrm{k}$ from a healthy donor (https://support.10xgenomics.com/single-cell-gene-expression/datasets/1.1.0/pbmc6k). After data quality controls, the read count tables of PBMC 4k (resp. PBMC 6k) contained 6,658 (resp. 5,169) genes and 3,815 (resp. 4,878) cells.

\section{Human small cell lung cancer with cisplatin treatments}

The data were obtained from circulating tumor cell-derived xenografts cultured with cisplatin treatments, which were generated from lung cancer patients (Stewart, et al., 2020). The data were produced with a 10x protocol using UMIs. The SRA files were 
downloaded from Gene Expression Omnibus (GEO) with accession codes GSE138474: GSM4104164, which is referenced in Stewart et al. (2020). SRA Toolkit version 2.10.8 was used to dump the FASTQ files. Cell Ranger version 3.1.0 was used to align the FASTQ files to the GRCh38-3.0.0 human reference genome and produce the single-cell transcriptome datasets. After controlling for data quality, the read count table contained 6,581 genes and 3,923 cells.

\section{Human pancreatic ductal adenocarcinoma}

The single-cell RNA sequencing (scRNA-seq) and spatial transcriptome (ST) data were obtained from PDAC patients using inDrop and ST protocols (Moncada, et al., 2020), respectively. The FASTQ files were downloaded from Gene Expression Omnibus (GEO) with accession codes GSE111672: GSM3036909, GSM3036910, GSM3036911, GSM3405527, GSM3405528, GSM3405529, and GSM3405530. Mapping of raw sequencing data from inDrop and ST protocols were processed using custom pipelines from https://github.com/flo-compbio/singlecell and https://github.com/jfnavarro/st_pipeline, respectively. Both pipelines used the parameters explained by Moncada et al. (2020). Prior to downstream analysis, we concatenated all the scRNA-seq datasets. After data quality controls, the read count table of the combined scRNA-seq dataset contained 5,893 genes and 2,051 cells, wherein the ST dataset contained 4,497 genes and 428 ST spots. ST data was imported and visualized using Spaniel (Queen, et al., 2019).

\section{Supplementary Note 4. Data preprocessing: quality control, normalization, and centering}

For all the scRNA-seq datasets, the low-quality genes and cells were removed by the following three steps: (i) removing the genes for which the number of non-zero expressing cells is less than a user-defined threshold; (ii) removing the cells whose read counts, number of genes expressed with non-zero read counts, and percent of reads mapped to mitochondrial genes are within user-defined ranges; and (iii) removing the genes for which the mean of the read counts is less than a user-defined threshold. See Chapters 2 and 3 in our tutorial (https://keita-iida.github.io/ASURAT_0.0.0.9001/index.html). 
After applying data quality controls, the data were normalized by bayNorm (Tang, et al., 2020), which attenuates technical biases with respect to zero inflation and variation of capture efficiencies between cells. The resulting inferred true count matrices were supplied to a log-transformation with a pseudo-count to attenuate the impact of dispersion in the counts for highly expressed genes. Finally, subtracting the sample mean from each row vector, we obtained the normalized-and-centered read count tables. See Chapter 4 in our tutorial (https://keitaiida.github.io/ASURAT_0.0.0.9001/index.html).

\section{Supplementary Note 5. Analysis of scRNA-seq datasets of PBMC 4k and 6k}

To compare the cell-type inference abilities of existing methods and ASURAT, we prepared two scRNA-seq datasets, namely PBMCs $4 \mathrm{k}$ and $6 \mathrm{k}$ (see Datasets). Subsequently, data quality controls and normalization by bayNorm were carefully performed for each dataset. See Chapters 2-4 in our tutorial (https://keitaiida.github.io/ASURAT_0.0.0.9001/index.html).

Using scran (version 1.18.7) (Lun, et al., 2016), we normalized the data using the functions quickCluster(), computeSumFactors(), and $\operatorname{logNormCounts(),~selected~highly~}$ variable genes using modelGeneVar() and getTopHVGs() based on a variance modeling with a gene-per-cell ratio of 0.2 (as suggested in a previous work (Cruz and Wishart, 2007)), and set the principal components using denoisePCA(). Cells were clustered using buildSNNGraph() and cluster_louvain(). Then, candidates of differentially expressed genes (DEGs) were detected using pairwiseTTests() and combineMarkers(), and DEGs were defined as genes with false discovery rates (FDRs) $<10^{-99}$ (T tests). According to the DEGs, we identified several different cell types by manually searching for marker genes in GeneCards version 5.2 (Stelzer, et al., 2016) as follows: B cells (resp. marker genes CD79A, MS4A1, IGHM), monocytes (S100A8, LYZ, CD14), NK or NKT cells (NKG7, GZMA, FGFBP2), and $\mathrm{T}$ cells $(M A L)$. See Chapter 13 in our tutorial (https://keita-iida.github.io/ASURAT_0.0.0.9001/index.html).

Using Seurat (version 4.0.2) (Hao, et al., 2021), we normalized the data using the function NormalizeData() with a log normalization (default), selected highly variable genes using 
FindVariableFeatures() based on a variance-stabilizing transformation with a gene-percell ratio of 0.2 (as suggested in previous work (Cruz and Wishart, 2007)), scaled and centered gene expression levels, and performed PCA. The principal components that explained $90 \%$ of the total variability were used for the computations of FindNeighbors(). Cells were clustered using FindClusters(). Then, candidates of DEGs were detected using FindAllMarkers() and DEGs were defined as genes with false discovery rates (FDRs)< $10^{-99}$ (Mann-Whitney $U$ tests). According to the DEGs, we identified several different cell types by manually searching for marker genes in GeneCards version 5.2 (Stelzer, et al., 2016) as follows: T cells (resp. marker genes TRAC, CD3D, IL32, TCF7, CD27), monocytes (S100A8, LYZ, CD14), B cells (CD79A, MS4A1, IGHM, VPREB3, BANK1), and NK or NKT cells ( $C D 3 D, N K G 7, G Z M A, F G F B P 2)$. Additionally, to automatically annotate the clustering results, we used the $\mathrm{R}$ function findmarkergenes() in the scCATCH (version 2.1) package (Shao, et al., 2020), which identified monocytes, B cells, and $\mathrm{T}$ cells. See Chapter 14 in our tutorial (https://keitaiida.github.io/ASURAT_0.0.0.9001/index.html).

Using Monocle 3 (version 1.0.0) (Trapnell, et al., 2014), we used the function preprocess_cds() under the default settings, in which data were normalized by a log transform with a pseudo-count of 1, scaled and centered in gene expression levels, and were subjected to PCA with the dimensionality of the reduced space set to 50. Cells were clustered by cluster_cells() using Uniform Manifold Approximation and Projection (UMAP) (McInnes and Healy, 2018). Then, candidate DEGs were detected using top_markers() and DEGs were defined as genes with false discovery rates (FDRs)< $10^{-99}$ (Monocle's marker significance tests). According to the DEGs, we identified several different cell types by manually searching for marker genes in GeneCards version 5.2 (Stelzer, et al., 2016) as follows: T cells (resp. marker genes $C D 3 D, T C F 7, C D 3 E$, IL32), monocytes (S100A8, LYZ, CD14), B cells (CD79A, CD79B, BANK1, MS4A1), and NK or NKT cells (GNLY, NKG7, GZMA). See Chapter 15 in our tutorial (https://keitaiida.github.io/ASURAT_0.0.0.9001/index.html).

Using SC3 (version 1.18.0) (Kiselev, et al., 2017), we performed the function runPCA() inputting log-normalized read count tables with a pseudo-count of 1 . Cells were clustered 
using sc3(), and reasonable numbers of clusters were manually determined by sc3_plot_markers(). Then, candidate DEGs were detected using get_marker_genes() and DEGs were defined as genes with false discovery rates $($ FDRs $)<10^{-99}$ (Kruskal-Wallis tests). According to the DEGs, we identified several different cell types by manually searching for marker genes in GeneCards version 5.2 (Stelzer, et al., 2016) as follows: NK or NKT cells (resp. marker genes GZMA, GZMB, GZMH, GZMK, GNLY), T cells (TRGC2, TCL1A), monocytes (GSN, LILRB4, S100A8, CD14, S100A12), and B cells (CD79A, CD79B, MS4A1, SPI1, LYN). See Chapter 16 in our tutorial (https://keitaiida.github.io/ASURAT_0.0.0.9001/index.html).

Using ASURAT, we created SSMs using the CO, GO, and KEGG DBs. After dimensionality reduction by PCA, cells were clustered by $k$-nearest neighbor (KNN) graph generation and Louvain algorithm using Seurat functions FindNeighbors() and FindClusters() (Hao, et al., 2021). Subsequently, separation indices (SIs) were computed for all the signs for a given cluster versus all the others, then cell types were identified by manually selecting significant signs with the larger values of SIs $>0.5$ (Figure 3). See Chapter 17 in our tutorial (https://keitaiida.github.io/ASURAT_0.0.0.9001/index.html).

\section{Supplementary Note 6. Analysis of an SCLC scRNA-seq dataset}

For the analysis of an SCLC scRNA-seq dataset, we began the Seurat workflow by normalizing data using the Seurat function NormalizeData() with a log normalization (default). Then, highly variable genes were selected by FindVariableFeatures() based on a variance stabilizing transformation with a gene-per-cell ratio of 0.2 (as suggested in previous work (Cruz and Wishart, 2007)). Then, data were scaled and centered by ScaleData(), and PCA was applied by $\operatorname{RunPCA}()$ with highly variable genes. Subsequently, a KNN graph was generated by FindNeighbors(), with the principal components that explain $90 \%$ of the total variability, and cells were clustered by FindClusters() with a Louvain algorithm. Additionally, cell cycle phases were inferred by CellCycleScoring() with cell cycle-related genes defined in the Seurat package. Finally, KEGG enrichment analysis was done by compareCluster() in clusterProfiler package (Yu, et al., 2012). See Chapter 14 in our tutorial (https://keita- 
iida.github.io/ASURAT_0.0.0.9001/index.html).

The ASURAT workflow started with the collection of DO, GO, and KEGG databases. First, we excluded functional gene sets including too few or too many genes. Next, we created multiple signs using a correlation graph-based decomposition. Then, we removed redundant signs with similar biological meanings using doSim() from the DOSE package (Yu, et al., 2015). We then created SSMs for DO, GO, and KEGG. Based on the SSM for DO, we performed a dimensionality reduction using the diffusion map and clustered cells using MERLoT (Parra, et al., 2019). Finally, we vertically concatenated all the SSMs, cell cycle phases inferred by Seurat, and expression matrix for characterizing individual cells from multiple biological aspects. The DEGs were identified using FindAllMarkers() in Seurat package. See Chapters 9-12 in our tutorial (https://keita-iida.github.io/ASURAT_0.0.0.9001/index.html).

\section{Supplementary Note 7. Limitations of the study}

To formulate signs, we used a correlation graph-based decomposition based on functional gene sets (FGSs) with thresholds set as positive and negative correlation coefficients (Figure 2), from which we obtained SCGs, VCGs, and WCGs. Although this method is intuitive and easy to use, such three-part decomposition might be insufficient in some cases. For example, one cannot divide the FGS for the DO term "lung small cell carcinoma" (DOID 5409) into more than three parts, while SCLC can be classified into at least four molecular subtypes (Schwendenwein, et al., 2021; Yatabe, 2020). Therefore, development of a more flexible method for dividing the correlation graphs is warranted.

Signs are derived from information in existing DBs. This inevitably introduces bias, such as the inherent incompleteness of the DBs and annotation bias; viz., some biological terms are associated with many genes, while others are associated with few (Gaudet and Dessimoz, 2017). To overcome this problem, one should monitor what signs are included during data processing (Figure 1a) and carefully tune the parameters to select reliable signs (Figure S1). Our R scripts help users perform this process. 


\section{References}

Andrews, T.S., et al. Tutorial: guidelines for the computational analysis of single-cell RNA sequencing data. Nat Protoc 2021;16(1):1-9.

Aran, D., et al. Reference-based analysis of lung single-cell sequencing reveals a transitional profibrotic macrophage. Nat Immunol 2019;20(2):163-172.

Balanis, N.G., et al. Pan-cancer Convergence to a Small-Cell Neuroendocrine Phenotype that Shares Susceptibilities with Hematological Malignancies. Cancer Cell 2019;36(1):17-34 e17.

Blondel, V.D., et al. Fast unfolding of communities in large networks. J Stat Mech-Theory E 2008:P10008.

Bodenhofer, U., Kothmeier, A. and Hochreiter, S. APCluster: an R package for affinity propagation clustering. Bioinformatics 2011;27(17):2463-2464.

Butler, A., et al. Integrating single-cell transcriptomic data across different conditions, technologies, and species. Nat Biotechnol 2018;36(5):411-420.

Cancer Genome Atlas Research, N., et al. Integrated genomic and molecular characterization of cervical cancer. Nature 2017;543(7645):378-384.

Cao, Y., Wang, X. and Peng, G. SCSA: A Cell Type Annotation Tool for Single-Cell RNA-seq Data. Front Genet 2020;11:490.

Chen, H.J., et al. Generation of pulmonary neuroendocrine cells and SCLC-like tumors from human embryonic stem cells. $J$ Exp Med 2019;216(3):674-687.

Chen, Z., et al. Ligand-receptor interaction atlas within and between tumor cells and T cells in lung adenocarcinoma. Int J Biol Sci 2020;16(12):2205-2219.

Coifman, R.R. and Lafon, S. Diffusion maps. Appl Comput Harmon A 2006;21:5-30.

Couper, P. A Student's Introduction to Geographical Thought: Theories, Philosophies, Methodologies. 2015.

Cruz, J.A. and Wishart, D.S. Applications of machine learning in cancer prediction and prognosis. Cancer Inform 2007;2:59-77.

De Simone, M., Rossetti, G. and Pagani, M. Single Cell T Cell Receptor Sequencing: Techniques and Future Challenges. Front Immunol 2018;9:1638.

Devitt, K., et al. Single-cell RNA sequencing reveals cell type-specific HPV expression in hyperplastic skin lesions. Virology 2019;537:14-19. 
Diehl, A.D., et al. The Cell Ontology 2016: enhanced content, modularization, and ontology interoperability. J Biomed Semantics 2016;7(1):44.

Dominguez, D., et al. A high-resolution transcriptome map of cell cycle reveals novel connections between periodic genes and cancer. Cell Res 2016;26(8):946-962.

Elosua-Bayes, M., et al. SPOTlight: seeded NMF regression to deconvolute spatial transcriptomics spots with single-cell transcriptomes. Nucleic Acids Res 2021;49(9):e50. Fan, J., et al. Characterizing transcriptional heterogeneity through pathway and gene set overdispersion analysis. Nat Methods 2016;13(3):241-244.

Gao, F., et al. DeepCC: a novel deep learning-based framework for cancer molecular subtype classification. Oncogenesis 2019;8(9):44.

Gaudet, P. and Dessimoz, C. Gene Ontology: Pitfalls, Biases, and Remedies. Methods Mol Biol 2017;1446:189-205.

Hao, Y., et al. Integrated analysis of multimodal single-cell data. Cell 2021;184(13):3573-3587 e3529.

Hyvarinen, A. Fast and robust fixed-point algorithms for independent component analysis. IEEE Trans Neural Netw 1999;10(3):626-634.

Ireland, A.S., et al. MYC Drives Temporal Evolution of Small Cell Lung Cancer Subtypes by Reprogramming Neuroendocrine Fate. Cancer Cell 2020;38(1):60-78 e12.

Ischenko, I., et al. KRAS drives immune evasion in a genetic model of pancreatic cancer. Nat Commun 2021;12(1):1482.

Jalili, M., et al. Exploring the Metabolic Heterogeneity of Cancers: A Benchmark Study of Context-Specific Models. J Pers Med 2021;11(6).

Kanehisa, M. and Goto, S. KEGG: kyoto encyclopedia of genes and genomes. Nucleic Acids Res 2000;28(1):27-30.

Kim, N., et al. Single-cell RNA sequencing demonstrates the molecular and cellular reprogramming of metastatic lung adenocarcinoma. Nat Commun 2020;11(1):2285.

Kiselev, V.Y., Andrews, T.S. and Hemberg, M. Challenges in unsupervised clustering of single-cell RNA-seq data. Nat Rev Genet 2019;20(5):273-282.

Kiselev, V.Y., et al. SC3: consensus clustering of single-cell RNA-seq data. Nat Methods 2017;14(5):483-486.

Kristiansen, G., et al. CD24 is an independent prognostic marker of survival in nonsmall cell lung cancer patients. Br J Cancer 2003;88(2):231-236. 
Kubota, S., et al. Dedifferentiation of neuroendocrine carcinoma of the uterine cervix in hypoxia. Biochem Biophys Res Commun 2020;524(2):398-404.

La Manno, G., et al. RNA velocity of single cells. Nature 2018;560(7719):494-498.

Lahnemann, D., et al. Eleven grand challenges in single-cell data science. Genome Biol 2020;21(1):31.

Lee, J., Hyeon, D.Y. and Hwang, D. Single-cell multiomics: technologies and data analysis methods. Exp Mol Med 2020;52(9):1428-1442.

Li, H., et al. Reference component analysis of single-cell transcriptomes elucidates cellular heterogeneity in human colorectal tumors. Nat Genet 2017;49(5):708-718.

Liu, X., et al. The reciprocal regulation between host tissue and immune cells in pancreatic ductal adenocarcinoma: new insights and therapeutic implications. Mol Cancer 2019;18(1):184.

Lowrance, R. and Wagner, R.A. An extension of the string-to-string correction problem. J Assoc Comput Mach 1975;22.

Luchini, C., et al. KRAS wild-type pancreatic ductal adenocarcinoma: molecular pathology and therapeutic opportunities. J Exp Clin Cancer Res 2020;39(1):227.

Lun, A.T., Bach, K. and Marioni, J.C. Pooling across cells to normalize single-cell RNA sequencing data with many zero counts. Genome Biol 2016;17:75.

Maynard, A., et al. Therapy-Induced Evolution of Human Lung Cancer Revealed by Single-Cell RNA Sequencing. Cell 2020;182(5):1232-1251 e1222.

McInnes, L. and Healy, J. UMAP: uniform manifold approximation and projection for dimension reduction. Preprint at https://arxiv.org/abs/1802.03426 2018.

McLeay, R.C. and Bailey, T.L. Motif Enrichment Analysis: a unified framework and an evaluation on ChIP data. BMC Bioinformatics 2010;11:165.

Moncada, R., et al. Integrating microarray-based spatial transcriptomics and single-cell RNA-seq reveals tissue architecture in pancreatic ductal adenocarcinomas. Nat Biotechnol 2020;38(3):333-342.

Mootha, V.K., et al. PGC-1alpha-responsive genes involved in oxidative phosphorylation are coordinately downregulated in human diabetes. Nat Genet 2003;34(3):267-273.

Morrison, R.E., Baptista, R. and Marzouk, Y. Beyond normality: Learning sparse probabilistic graphical models in the non-Gaussian setting. Adv Neur In 2017;30. 
Muller-Hubenthal, B., et al. Tumour Biology: tumour-associated inflammation versus antitumor immunity. Anticancer Res 2009;29(11):4795-4805.

Murtagh, F. and Legendre, P. Ward's hierarchical agglomerative clustering method: which algorithms implement Ward's criterion? J Classif 2014;31:274-295.

Parra, R.G., et al. Reconstructing complex lineage trees from scRNA-seq data using MERLoT. Nucleic Acids Res 2019;47(17):8961-8974.

Pasquini, G., et al. Automated methods for cell type annotation on scRNA-seq data. Comput Struct Biotechnol J 2021;19:961-969.

Queen, R., et al. Spaniel: analysis and interactive sharing of Spatial Transcriptomics data. bioRxiv 2019.

Reimand, J., et al. Pathway enrichment analysis and visualization of omics data using g:Profiler, GSEA, Cytoscape and EnrichmentMap. Nat Protoc 2019;14(2):482-517.

Rempala, G.A., Seweryn, M. and Ignatowicz, L. Model for comparative analysis of antigen receptor repertoires. $J$ Theor Biol 2011;269(1):1-15.

Schubert, E. and Rousseeuw, P.J. Faster k-Medoids Clustering: Improving the PAM, CLARA, and CLARANS Algorithms. SISAP 2020 2019:171-187.

Schwendenwein, A., et al. Molecular profiles of small cell lung cancer subtypes: therapeutic implications. Mol Ther Oncolytics 2021;20:470-483.

Shao, X., et al. scCATCH: Automatic Annotation on Cell Types of Clusters from SingleCell RNA Sequencing Data. iScience 2020;23(3):100882.

Stelzer, G., et al. The GeneCards Suite: From Gene Data Mining to Disease Genome Sequence Analyses. Curr Protoc Bioinformatics 2016;54:1 30 31-31 3033.

Stewart, C.A., et al. Single-cell analyses reveal increased intratumoral heterogeneity after the onset of therapy resistance in small-cell lung cancer. Nat Cancer 2020;1:423-436.

Subramanian, A., et al. Gene set enrichment analysis: a knowledge-based approach for interpreting genome-wide expression profiles. Proc Natl Acad Sci $U$ S A 2005;102(43):15545-15550.

Tang, W., et al. bayNorm: Bayesian gene expression recovery, imputation and normalization for single-cell RNA-sequencing data. Bioinformatics 2020;36(4):11741181.

Trapnell, C., et al. The dynamics and regulators of cell fate decisions are revealed by pseudotemporal ordering of single cells. Nat Biotechnol 2014;32(4):381-386. 
Villani, A.C., et al. Single-cell RNA-seq reveals new types of human blood dendritic cells, monocytes, and progenitors. Science 2017;356(6335).

Wagner, F.Y., I. Moana: A robust and scalable cell type classification framework for single-cell RNA-Seq data. bioRxiv 2018.

Wooten, D.J., et al. Systems-level network modeling of Small Cell Lung Cancer subtypes identifies master regulators and destabilizers. PLoS Comput Biol 2019;15(10):e1007343. Yatabe, Y. Reassessing the SCLC Subtypes. J Thorac Oncol 2020;15(12):1819-1822.

$\mathrm{Yu}, \mathrm{G}$., et al. GOSemSim: an R package for measuring semantic similarity among GO terms and gene products. Bioinformatics 2010;26(7):976-978.

$\mathrm{Yu}, \mathrm{G}$., et al. clusterProfiler: an R package for comparing biological themes among gene clusters. OMICS 2012;16(5):284-287.

$\mathrm{Yu}, \mathrm{G}$., et al. DOSE: an R/Bioconductor package for disease ontology semantic and enrichment analysis. Bioinformatics 2015;31(4):608-609.

Zhang, A.W., et al. Probabilistic cell-type assignment of single-cell RNA-seq for tumor microenvironment profiling. Nat Methods 2019;16(10):1007-1015.

Zhang, W., et al. PIRD: Pan Immune Repertoire Database. Bioinformatics 2020;36(3):897-903.

Zhang, X., et al. CellMarker: a manually curated resource of cell markers in human and mouse. Nucleic Acids Res 2019;47(D1):D721-D728. 